\title{
Taking silicon photonics modulators to a higher performance level: state-of-the-art and a review of new technologies
}

\author{
Abdul Rahim $\odot,{ }^{a, b, \star}$ Artur Hermans $\odot,{ }^{a, b}$ Benjamin Wohlfeil, ${ }^{c}$ Despoina Petousi, ${ }^{c}$ Bart Kuyken, ${ }^{a, b}$ \\ Dries Van Thourhout, ${ }^{a, b}$ and Roel Baets ${ }^{a, b, *}$ \\ ${ }^{a}$ Ghent University, Photonics Research Group, Department of Information Technology, Ghent, Belgium \\ ${ }^{b}$ Ghent University, IMEC and Center for Nano- and Biophotonics, Ghent, Belgium \\ ${ }^{\mathrm{C} A D V A}$ Optical Networking, Berlin, Germany
}

\begin{abstract}
Optical links are moving to higher and higher transmission speeds while shrinking to shorter and shorter ranges where optical links are envisaged even at the chip scale. The scaling in data speed and span of the optical links demands modulators to be concurrently performant and cost-effective. Silicon photonics (SiPh), a photonic integrated circuit technology that leverages the fabrication sophistication of complementary metal-oxide-semiconductor technology, is well-positioned to deliver the performance, price, and manufacturing volume for the high-speed modulators of future optical communication links. SiPh has relied on the plasma dispersion effect, either in injection, depletion, or accumulation mode, to demonstrate efficient high-speed modulators. The high-speed plasma dispersion silicon modulators have been commercially deployed and have demonstrated excellent performance. Recent years have seen a paradigm shift where the integration of various electro-refractive and electro-absorptive materials has opened up additional routes toward performant SiPh modulators. These modulators are in the early years of their development. They promise to extend the performance beyond the limits set by the physical properties of silicon. The focus of our study is to provide a comprehensive review of contemporary (i.e., plasma dispersion modulators) and new modulator implementations that involve the integration of novel materials with SiPh.
\end{abstract}

Keywords: high-speed modulators; silicon photonics; plasma dispersion effect; ferroelectrics; graphene; III-V on Si; organic (electro-optic) materials.

Received Sep. 24, 2020; revised manuscript received Jan. 19, 2021; accepted for publication Mar. 10, 2021; published online Apr. 29, 2021.

(C) The Authors. Published by SPIE and CLP under a Creative Commons Attribution 4.0 Unported License. Distribution or reproduction of this work in whole or in part requires full attribution of the original publication, including its DOI.

[DOI: 10.1117/1.AP.3.2.024003]

\section{Introduction}

High-speed transceivers for short-reach (few $\mathrm{cm}$ to few tens of $\mathrm{km}$ ) and long-haul optical communication links require high-performance modulators in their transmitter optical subassembly. ${ }^{1-3}$ The key performance parameters for an efficient modulator are (a) high modulation efficiency ${ }^{4-9}$ in amplitude or phase by a drive signal that is compliant with the complementary metal-oxide-semiconductor (CMOS) circuitry ${ }^{5,6}$ [for phase modulators, the modulation efficiency is defined as the product

*Address all correspondence to Abdul Rahim, abdul.rahim@ugent.be; Roel Baets, roel.baets@ugent.be of voltage $V_{\pi}$ applied to the phase shifter to achieve $\pi$ phase shift and the length $L$ of the phase shifter-a smaller $V_{\pi} \cdot L$ represents higher modulation efficiency; for amplitude modulation, the ratio of extinction ratio (ER) to an applied voltage for a given length of the modulator represents the modulation efficiency and large values are desired]; (b) low insertion loss (IL), ${ }^{10-13}$ both low loss in the active and passive parts of the modulator; (c) tens of gigabits per second $(\mathrm{Gb} / \mathrm{s})^{14-17}$ modulation speed to support the future capacity demands of the optical communication networks; (d) energy consumption to be as low as a few tens of $\mathrm{fJ} / \mathrm{bit}^{18-20}$ (e) large ER-typically $\sim 4 \mathrm{~dB}$ for $\sim 2 \mathrm{~km}$ transmission links and $\sim 8 \mathrm{~dB}$ for tens of $\mathrm{km}$ long transmission linksto ensure high optical signal-to-noise ratio for maintaining a low 
bit error rate ${ }^{21-25}$ (f) low chirp ${ }^{26-28}$ to realize higher-order modulation schemes ${ }^{27-29}$ and to enhance dispersion tolerance of transmission links; $;^{26,30}$ and $(\mathrm{g})$ compact $\operatorname{size}^{9,12,31-33}$ to enable high-integration density. ${ }^{1,2,21,34-36}$

Generally, the modulator figure of merit (FoM) captures the performance of a modulator. In the literature, a variety of definitions for modulator FoM exist. ${ }^{1,2,21,37,38}$ In its simplest form, $V_{\pi} \cdot L$ represents the FoM for a phase modulator. $V_{\pi} \cdot L$. $\alpha$ is a phase modulator FoM that takes the phase shifter loss $\alpha$ into account. A phase modulator FoM given as $f_{c} \cdot\left[C_{L} \cdot\left(V_{\pi} \cdot L\right)^{2} \cdot \alpha / 8\right]^{-1}$, where $f_{c}$ represents the phase shifter cutoff frequency and $C_{L}$ represents the phase shifter capacitance, relates the FoM in terms of modulator bandwidth, loss, and energy per bit. ${ }^{2,4,21}$ The ratio ER/IL gives the FoM for amplitude modulators. ${ }^{20,38}$ Dynamic optical modulation amplitude is a dynamic FoM of a modulator, as it takes modulator parameters and the modulation format into account. ${ }^{37}$

High-index-contrast silicon photonics $(\mathrm{SiPh})$ provides dense integration of complex photonic functionalities, such as highspeed modulators, using technology and process toolsets from CMOS electronics. ${ }^{39}$ Other photonic integrated circuit (PIC) platforms such as indium phosphide, ${ }^{40-42}$ which typically relies on $100 \mathrm{~mm}$ wafers, and lithium niobate $\left(\mathrm{LiNbO}_{3}\right),{ }^{43}$ which commonly relies on $150 \mathrm{~mm}$ wafers, are fabricated in customized fabs. $\mathrm{SiPh}$ integrated circuits are typically fabricated on 200 or $300 \mathrm{~mm}$ wafers. The large wafer size allows for a large number of compact dies per wafer at low-cost scaling to large commercial volumes in existing CMOS commercial fabs and R\&D pilot lines. ${ }^{39,44-48} \mathrm{SiPh}$ modulators promise to provide the best price-performance ratio and possible routes for monolithic integration of electronics and photonics. ${ }^{2,21,22,39,49}$ The commercial launch of SiPh-enabled transceivers is a testament to the promise of $\mathrm{SiPh}$ as a photonic integration technology to deliver efficient high-speed modulators. ${ }^{3,44-48,50-52}$

The push for performant high-speed modulators stems from the ever booming growth of internet traffic. This growth has driven the modern data centers (DCs) into the so-called "Zettabyte Era," where the annual global traffic is expected to exceed 2.2 zettabytes/year. This value corresponds to a threefold increase in just five years. The biggest contribution to this huge traffic originates from data moving inside the DCs over inter-DC connections. ${ }^{53}$ Nonetheless, a remarkable increase in data exchanged between different DCs is also reported within the last few years (inter-DC connections). Future massive-scale DCs are expected to spread widely across the globe in order to meet the enormous bandwidth demands. An increase in baud rate imposes itself, yet at an even lower power consumption per bit. Consequently, crucial optimization of the intra- and inter-data center interconnects (DCIs) - where high-speed modulators are a key building block-is needed. More specifically:

- The inter-DCI landscape is dominated by coherent dense wavelength division multiplexing optics for reaching high capacities per single wavelength, currently targeting up to $38.4 \mathrm{~Tb} / \mathrm{s}$ aggregate capacity in a distance between 40 and 1000 s of kilometers with data rates of currently $400 \mathrm{~Gb} / \mathrm{s}$ to $600 \mathrm{~Gb} / \mathrm{s}$ (64 Gbaud) per wavelength. By 2025, data rates are expected to double ${ }^{54}$ with symbol rates exceeding 100 Gbaud. These values set strict requirements regarding the transmitter performance, primarily in terms of bandwidth. Regarding power consumption, pluggable modules such as $\mathrm{C}$ form factor pluggable (CFP) and CFP2 used for current
inter-DCIs are limited to a maximum power consumption of around $20 \mathrm{~W}$ in total, and new form factors such as octal small form factor pluggable or quad small form factor pluggabledouble density are specified to an even lower maximum power consumption. This means that the modulator and driver power consumption should be carefully optimized. The most widely used architecture for inter-DCIs is the Mach-Zehnder modulator (MZM) in in-phase, quadrature configuration, whereby MZMs are nested to modulate the in-phase and quadrature component of the signal. ${ }^{55,56}$ For demonstrating high-order quadrature amplitude modulation (QAM), it is extremely critical for the MZM to show a linear intensity dependence with voltage (low chirp) to minimize the error vector magnitude. ${ }^{27}$ Furthermore, the need for more bandwidth in wavelength division multiplexing systems requires broadband and tunable devices, increasing the need to optimize components for use in optical bands beyond the C-band (1.53 to $1.565 \mu \mathrm{m}$ ), i.e., L-band (1.565 to $1.625 \mu \mathrm{m}$ ) and/or S-band (1.46 to $1.53 \mu \mathrm{m}$ ).

- Due to the shorter distance requirements of up to $2 \mathrm{~km}$ (FR) or up to $10 \mathrm{~km}$ (LR), the intra-DCI applications are mainly using multi-channel 56 Gbaud, four-level pulse amplitude modulation (PAM-4). As a result, the transmitter requirements in this case are more relaxed in terms of linearity, but a high dynamic ER $\left(\mathrm{ER}>4.5 \mathrm{~dB}^{57}\right)$ is necessary in order to achieve multi-level modulation. In this case, QSFP form factors require a maximum total power consumption of $5.5 \mathrm{~W}$. Mainly because of the very strict power consumption requirements, up to now, vertical-cavity surface-emitting laser (VCSEL)-based transceivers and parallel fiber transmission have been used inside DCs, especially on short reaches. However, with the massive increase of traffic, there has been a continuous interest to replace VCSELs with next generation ultra-compact devices that will provide even lower cost-energy consumption figures. With the next generations of intra-DCI transceivers aiming for data rates of $800 \mathrm{~Gb} / \mathrm{s}$ and beyond, a potential move of coherent technology into the domain of intensity modulation and direct detection may be seen, as scaling of intensity modulated signals to higher capacity is increasingly challenging. ${ }^{58}$ However, to meet the stringent power consumption target for intra-DCI transceivers, lightweight digital signal processing, lower power consumption components, and potentially a reduced coherent system will be required.

For both intra- and inter-DCIs, $\mathrm{SiPh}$ appears to be a promising technology platform, ${ }^{59,60}$ mainly because of the dense integration of photonics ${ }^{59,60}$ while minimizing the overall costpower efficiency budget. ${ }^{18,19,39}$ Currently, the plasma dispersion effect, which is based on the movement (injection, $7,37,61-67$ accumulation, ${ }^{12,68-70}$ or depletion ${ }^{9,13,14,16,22,32,33,71-87}$ ) of carriers to induce a refractive index change in a silicon waveguide, is the most widely deployed phenomenon to implement high-speed modulators in SiPh.,7-19,22,32,33,37,61-69,69-71,71-121 Plasma dispersion modulators in $\mathrm{SiPh}$ have successfully demonstrated operations of 90 (Ref. 87) and $100 \mathrm{~Gb} / \mathrm{s}^{122,123}$ for the on-off keying (OOK) modulation scheme. Furthermore, various plasma dispersion modulators implementations for advanced modulation schemes such as QPSK, ${ }^{55,56,124}$ QAM, ${ }^{125,126}$ PAM-4, ${ }^{29,52,127}$ and PAM-8 ${ }^{115}$ also exist.

The plasma dispersion effect is a weak electro-refractive effect. ${ }^{128} \mathrm{~A} 10^{17} \mathrm{~cm}^{-3}$ change in carrier concentration introduces an index change of $\Delta n \sim 10^{-4}$. Furthermore, the phase modulation provided by the plasma dispersion effect is always 
associated with spurious amplitude modulation. ${ }^{21,26}$ Unlike Pockels modulators, plasma dispersion modulators do not have a linear dependence of voltage and refractive index change $\Delta n$ (thus, they show chirp), which makes them a less promising candidate for cases that require pure phase modulation. $26,55,56,124,125,129$ Nevertheless, R\&D efforts have resulted in the performance of $\mathrm{SiPh}$ plasma dispersion modulators that is comparable with other photonic integration technologies. ${ }^{27}$

Typically, plasma dispersion modulators rely on interferometric structures [such as the Mach-Zehnder interferometer (MZI) $^{13,19,78,84,108,118}$ or optical cavities, ${ }^{9,12,32,33,100,115}$ ] for the translation of phase modulation into amplitude modulation. The carrier depletion-based interferometric plasma dispersion modulators, such as MZMs, are large in size (typically a phase shifter length of a few mm). ${ }^{21,22}$ Injection or accumulation of carriers offers higher efficiency plasma dispersion effect phase shifters, enabling compact interferometric modulators. ${ }^{37,65}$ The implementations of plasma dispersion high-speed modulators involving optical cavities [such as ring resonators (RRs)] result in compact devices (typically a few hundreds of $\mu \mathrm{m}^{2}$ ).,12,32,33 However, these RR-based devices suffer from high temperature and fabrication sensitivity. ${ }^{130,131}$ Literature reports solving these problems to a certain extent through smart design strategies and technological advancements. ${ }^{132}$ Nonetheless, the limited operational optical bandwidth of the ring modulator (RM) is intrinsic to them.

It is a daunting task to deliver the future demands for single lane 100 Gbaud and beyond data rates in an energy-efficient manner. Recently, there have been a growing number of efforts to integrate electro-optic materials with a strong Pockels effect on SiPh platforms. The integration of such electro-optic materials promises to provide additional routes to upscale the performance of SiPh modulators to deliver the future demands for single lane 100 Gbaud and beyond data rates in an energyefficient manner. Table 1 shows a rich landscape of $\mathrm{SiPh}$ modulators, where $\mathrm{SiPh}$ is incorporating a variety of other materials for implementing performant high-speed modulators. Modulation of amplitude by the Franz-Keldysh (FK) effect, ${ }^{20,38,128,133-140}$ quantum-confined Stark (QCS) effect, ${ }^{141-152}$ and electrical gating, ${ }^{153-160}$ or the modulation of phase by employing the plasma dispersion effect, ${ }^{161-166}$ Pockels effect, ${ }^{167-192,192-200}$ and inter-band transitions, ${ }^{201-208}$ is feasible either directly in silicon $^{20,31,38,133-139,143}$ or by integrating a variety of efficient electro-optic materials with SiPh platforms. ${ }^{161,172,182,184,202}$ The integration of new materials into SiPh has to counter various challenges. Invariably, any new material integration requires new technology and process development that allows for low-loss integration with the $\mathrm{SiPh}$ platform. The integration process must ensure high material quality. The introduction of defects in the integration process downgrades the yield of the PIC manufacturing process. Consequently, the integration of new materials always adds to manufacturing costs. Materials allowing waferscale integration and the ones for which the fab can leverage from the existing learning curve of introduction into other platforms reduce the financial impact and technological complexities, and they are thus preferred.

The electrical driving of the modulator comprises an electrode configuration and the driving scheme (such as single-ended drive with dual-arm push-pull, differential drive with dualarm push-pull, differential drive with dual-arm push-pull with shared drive, or dual-differential drive with dual-arm push-pull) to feed the electrodes. ${ }^{25}$ Generally, the length of the phase shifter determines the electrode configuration. ${ }^{209}$ Modulators with a short phase shifter length (phase shifter length $<\lambda_{\mathrm{RF}} / 10$ ) ) $^{2,25}$ use lumped electrodes. ${ }^{209,210}$ Lumped driving is applicable to modulators comprising interferometric structures, ${ }^{65,210,211}$ as well as

Table 1 Prominent approaches for high-speed modulation in SiPh. The modulator implementations in SiPh use a variety of physical phenomenons, materials, optical architectures, and driver architectures.

The landscape of high-speed modulators in silicon photonics

\begin{tabular}{|c|c|c|c|c|}
\hline Modulation & Operating principle & Platform & $\begin{array}{l}\text { Reported optical } \\
\text { implementation }\end{array}$ & $\begin{array}{l}\text { Reported driver } \\
\text { implementation }\end{array}$ \\
\hline \multirow[t]{7}{*}{ Phase } & $\begin{array}{l}\text { Plasma dispersion effect by carrier } \\
\text { (a) Injection } \\
\text { (b) Accumulation } \\
\text { (c) Depletion }\end{array}$ & Silicon & $\begin{array}{l}\text { MZI, michelson, resonators } \\
\text { (ring, disk, ph. crystal, } \\
\text { Fabry-Perot), slow-light } \\
\text { structure, Bragg reflectors }\end{array}$ & $\begin{array}{l}\text { Lumped, traveling } \\
\text { wave (TW), } \\
\text { segmented }\end{array}$ \\
\hline & Pockels effect & $\mathrm{LiNbO}_{3}$ on silicon & $\mathrm{MZI}$ & Lumped, TW \\
\hline & & Organics on silicon & $\mathrm{MZI}$, ring resonator & Lumped, TW \\
\hline & & BTO on silicon & $\mathrm{MZI}$, ring resonator & Lumped, TW \\
\hline & & PZT on silicon & $\mathrm{MZI}$, ring resonator & Lumped \\
\hline & Interband transitions & 2D materials on silicon & $\mathrm{MZI}$, ring resonator & Lumped, TW \\
\hline & $\begin{array}{l}\text { Carrier accumulations/carrier } \\
\text { depletion+Franz-Keldysh effect }\end{array}$ & III-V on silicon & $\mathrm{MZI}$, ring resonator & Lumped, TW \\
\hline \multirow[t]{4}{*}{ Amplitude } & Franz-Keldysh effect & Silicon-germanium & Waveguide, MZI & Lumped \\
\hline & Quantum confined Stark effect & Ge-Si-Ge quantum wells & $\begin{array}{l}\text { Waveguide, } \\
\text { Fabry-Perot cavity }\end{array}$ & Lumped \\
\hline & Electrical gating & 2D materials on silicon & Waveguide & Lumped \\
\hline & Quantum confined Stark effect & III-V on silicon & Waveguide & Lumped \\
\hline
\end{tabular}


cavity-based structures ${ }^{9,12,62,70,115}$ and slow-light structures. ${ }^{112,113}$ With lumped electrodes, the applied voltage is approximately identical across the entire length of the phase shifter. Lumped driving does not necessarily require a matched termination of the driving electrodes. With unterminated lumped driving, the phase shifter's capacitive load determines the energy consumption per bit $\left(E_{\mathrm{bit}}\right)$ for a modulator. Typically, the bandwidth of lumped electrode phase shifter driving is limited by its impedance (intrinsic, parasitic, and the one contributed by the driving circuitry) and the time taken by the photons to transit through the phase shifter. The latter effect is more pronounced for the slow-light modulators. ${ }^{113,114}$

The traveling wave (TW) is the most common driving scheme for carrier depletion plasma dispersion MZMs. ${ }^{2,4,25,210,212}$ In this scheme, a single driver drives the entire electrode of the phase shifter. ${ }^{25,212}$ The TW electrode typically comprises a coplanar waveguide transmission line. It terminates with a resistive impedance matched to the wave impedance of the electrode. The RF power applied to the TW electrode is consumed by the RF losses in the TW, capacitive loading by the phase shifter, and the matched termination. The matched resistance prevents electrical reflections and consequent interference with the electrical signal stream. But, it also increases the power consumption of the TW electrode as a fraction of the total input RF power is always consumed by the matched termination. Generally, TW driving schemes are six to eight times more energy-hungry than the lumped driving., ${ }^{2,4}$ The detrimental factors impacting the bandwidth of the modulators with TW driving are: (a) RC time constant of the phase shifter; (b) walk-off (phase mismatch due to mismatch between the propagation speed of the electrical signal in the transmission line and the optical signal in the waveguide) between the electrical and optical signals; and (c) RFlosses in the TW electrode.

The segmented topology with distributed driving comprises a series of short phase shifters, which are individually driven by lumped electrodes. ${ }^{6,22,66}$ Segmentation of the phase shifter with distributed driving avoids the RF attenuation due to a long transmission line while enabling the same voltage swing across the whole length of the phase shifter. ${ }^{212}$ As compared to the TW architecture, the segmented electrodes require smallersize voltage drivers. ${ }^{25}$ The precise delivery of the electrical signal to a phase shifter segment requires time synchronization with the transit time of light in that segment, which requires timing control circuitry for precise delivery of the electrical signal to each segment. This additional overhead undermines the energy efficiency of the segmented drivers and adds to the driver complexity. The number of phase shifter segments and their length determine the modulator bandwidth and its power efficiency. Typically, segmented electrodes with distributed drivers require tighter connectivity between the electronic drivers and the phase shifter segments to preserve the signal integrity and prevent parasitic effects. Therefore, this driving scheme demands either monolithic integration of electronics and photonics or flip-chip bonding photonic and electronic chips through an array of microbumps. Electrical driving plays a crucial role in defining the performance (primarily the power consumption and bandwidth) of high-speed phase modulators. ${ }^{2,122,210}$ In the early years, most of the high-speed modulator demonstrations focused on the optimization of modulator performance in isolation from the driver design. ${ }^{122}$ Recent demonstrations of high-speed modulators have established that concurrent co-design and co-optimization of electrical driving and photonic phase shifter design ${ }^{25,52,122}$ are a key to extracting the best performance for all modulator performance attributes..$^{52,213}$ This holds for modulators involving monolithic or hybrid integration with electronics. ${ }^{25,122,213}$

This paper aims to provide a comprehensive review of the new technologies that provide additional, viable routes to enhance the performance of $\mathrm{SiPh}$ modulators. These new technologies involve the integration of materials with high electro-optic coefficients with $\mathrm{SiPh}$ platforms. As a result, they promise to deliver modulator performance that is required to meet the blistering surge in transmission rates for the optical links. This paper also compares these new technologies against the current mainstream $\mathrm{SiPh}$ modulator implementations. A comprehensive coverage in full detail is very challenging and difficult to be performed in a paper with a limited page number. Therefore, we will limit the discussion in this paper to some of the most important and relevant implementations of high-speed modulators in $\mathrm{SiPh}$. It is important to note that the $\mathrm{SiPh}$ modulators have been used to modulate signals by means of advanced modulation schemes such as QPSK, ${ }^{55,56,124}$ QAM, ${ }^{125,126}$ PAM-4, ${ }^{29,52,127}$ and PAM-8. ${ }^{115}$ However, in this paper, the operating speed is quoted only for OOK. Furthermore, the discussion in this paper is limited to C-band and O-band implementations of $\mathrm{SiPh}$ modulators for telecommunication and data communication applications; though $\mathrm{SiPh}$ modulators are also reported for mid-infrared (mid-IR) wavelengths ${ }^{214,215}$ and for non-telecom/ datacom applications. ${ }^{215}$

\section{State-of-the-Art of Contemporary Silicon Photonic High-Speed Modulators}

Research to develop SiPh modulators dates back to the era when it was still a niche choice for photonic integration. ${ }^{61,64,88-90,216}$ Unstrained silicon has a fundamentally zero linear electro-optic effect (the Pockels effect, used in traditional $\mathrm{LiNbO}_{3}$ modulators) due to its crystallographic structure (i.e., centrosymmetric crystal lattice), albeit strained silicon is investigated for highspeed all-silicon modulation. ${ }^{217-219}$ This intrinsic property of Si directed the researchers to investigate other optical phenomenons, such as the plasma dispersion effect to realize highperformance modulators in $\mathrm{SiPh}$. This section provides a summary of the plasma dispersion modulators to implement $\mathrm{SiPh}$ high-speed modulators.

\subsection{High-Speed Modulators Using the Plasma Dispersion Effect}

The refractive index change by the plasma dispersion effect in silicon is larger than the change by the Kerr ${ }^{61,220}$ and FK effects. ${ }^{31,63,141}$ The plasma dispersion effect is broadband going all the way from telecom to mid-IR wavelengths ${ }^{221}$ and is relatively temperature independent. ${ }^{222-225}$ Furthermore, its implementation is inherently simple, using standard CMOS processing technology. ${ }^{81}$ All of these factors made the plasma dispersion effect one of the current mainstream mechanisms to implement efficient high-speed modulators in $\mathrm{SiPh}^{2,21,35,49}$ Years of development, led by both industry and academic players, have resulted in the demonstration of plasma dispersion effect-based phase modulators providing losses of $<10 \mathrm{~dB} / \mathrm{cm}^{71,80,116,118,121}$ phase modulation efficiencies $V_{\pi} \cdot L<0.3 \mathrm{~V} \cdot \mathrm{cm},{ }^{5,69,104}$ energy consumption as low as tens of $\mathrm{fJ} / \mathrm{bit},{ }^{18,99,110,111,115}$ and data rates reaching $100 \mathrm{~Gb} / \mathrm{s}^{14,87,122,123}$ for OOK modulation with minimal 
influence of temperature on the performance of the plasma dispersion modulators (III-V modulators rely on the QCS effect and are influenced by temperature). It is important to note that all of these specs are not provided simultaneously by a single plasma dispersion modulation implementation. ${ }^{21}$

Plasma dispersion is an electro-refractive effect. The change of free carrier concentration by the movement of charge carriers into or out of a waveguide results in phase modulation of the optical signal. ${ }^{21,49,61}$ Phase modulation is translated into intensity modulation by embedding the phase modulator into MZIs, ${ }^{15-17,77,79,108,109}$ RRs, ${ }^{18,32,33,62,110,111,119}$ Bragg reflectors, ${ }^{88}$ Michelson interferometers, ${ }^{8,121}$ photonic crystal cavities, ${ }^{98,112-114}$ and Fabry-Perot cavities. ${ }^{100}$ The three prominent schemes to introduce change in the free carrier concentration are (a) the injection of minority carriers ${ }^{7,37,61-67}$ by forward biasing a PIN junction; (b) the accumulation of majority carriers ${ }^{12,68,69,69,70}$ of opposing polarity across an insulating section in a waveguide; (c) the depletion of majority carriers ${ }^{9,13,14,16,22,32,33,71,71-87}$ from a PN junction by reversely biasing it.

Carrier injection is the most efficient plasma dispersionbased modulation scheme. ${ }^{7,8,37,98}$ Most of the early demonstrations of high-speed modulators relied on carrier injection of minority carriers by forward biasing a PIN junction built along a waveguide. ${ }^{63,89,90,93,216,226}$ The carrier injection-based modulators provide a high modulation efficiency due to their large diffusion capacitance (typically $\sim 10 \mathrm{pF}$ ) when implemented on a waveguide with a small cross section with stronger confinement. ${ }^{737,65-67,91-94}$ Carrier injection-based modulators are reported using lateral $\mathrm{PIN}^{63,65,67,92}$ and vertical PIN configurations (see Fig. 1). ${ }^{63,89,93,216,226}$ Owing to the feasibility for a short phase shifter $(\sim$ a few hundreds of $\mu \mathrm{m})$, carrier injection modulators typically use lumped driving schemes. ${ }^{7,65,67}$ The use of pre-emphasized electrical signaling schemes, in which a fraction of the electrical driver signal has a voltage $\gg V_{\pi}$ of the modulator, boosts the limited speeds of carrier injection-based modulators ${ }^{67,95-97}$ with the obvious ramification of increased power consumption. Recent years have seen the development of passive equalization techniques to boost the high-speed operation of the carrier injection modulators. ${ }^{65,67}$ The studies have shown that the factors that limit the speed of the carrier injection-based modulation are (a) recombination time of the injected electron-hole pairs and (b) the sum of the electrical driver output resistance and the bulk resistance of the p- and n-doped regions. ${ }^{7,65,67}$ The slower of these two effects determines the speed limit of the carrier injection modulators ${ }^{67}$ Practically, it is possible to improve the bandwidth of carrier injection modulators by embedding an $R_{E} C_{E}$ passive equalizer such that $R_{E} C_{E}=R_{F} C_{F}$ and $C_{F} \gg C_{E}$, where $R_{E, F}$ and $C_{E, F}$ are the equalizer and the forward resistance and the capacitance, respectively. ${ }^{7,65,91}$ Current state-of-the-art carrier injection modulators use this passive equalization to demonstrate $>20 \mathrm{~Gb} / \mathrm{s}$ operation. ${ }^{7,65}$ Recently, 70 Gbaud operation of a carrier injection MZM was enabled by passive RC equalization. ${ }^{7}$ The modulator has a $37-\mathrm{GHz} 3-\mathrm{dB}$ bandwidth using a $0.25-\mathrm{mm}$ long-phase shifter having $\sim 28 \mathrm{~dB} / \mathrm{cm}$ loss and a $V_{\pi} \cdot L$ of $2 \mathrm{~V} \cdot \mathrm{cm}$. ${ }^{7}$ One of the best modulation efficiencies of $0.274 \mathrm{~V} \cdot \mathrm{cm}$ has

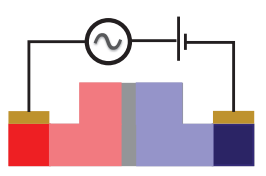

Carrier injection

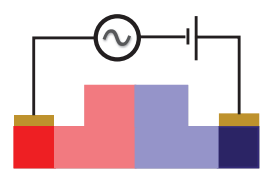

Carrier depletion

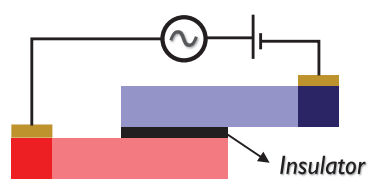

Carrier accumulation

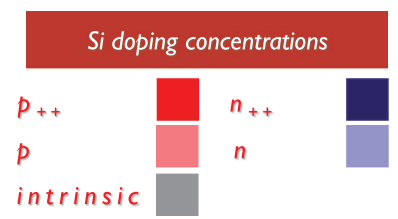

Baseline configurations of carrier injection (left), carrier depletion (center), and carrier accumulation phase shifters using plasma dispersion effect

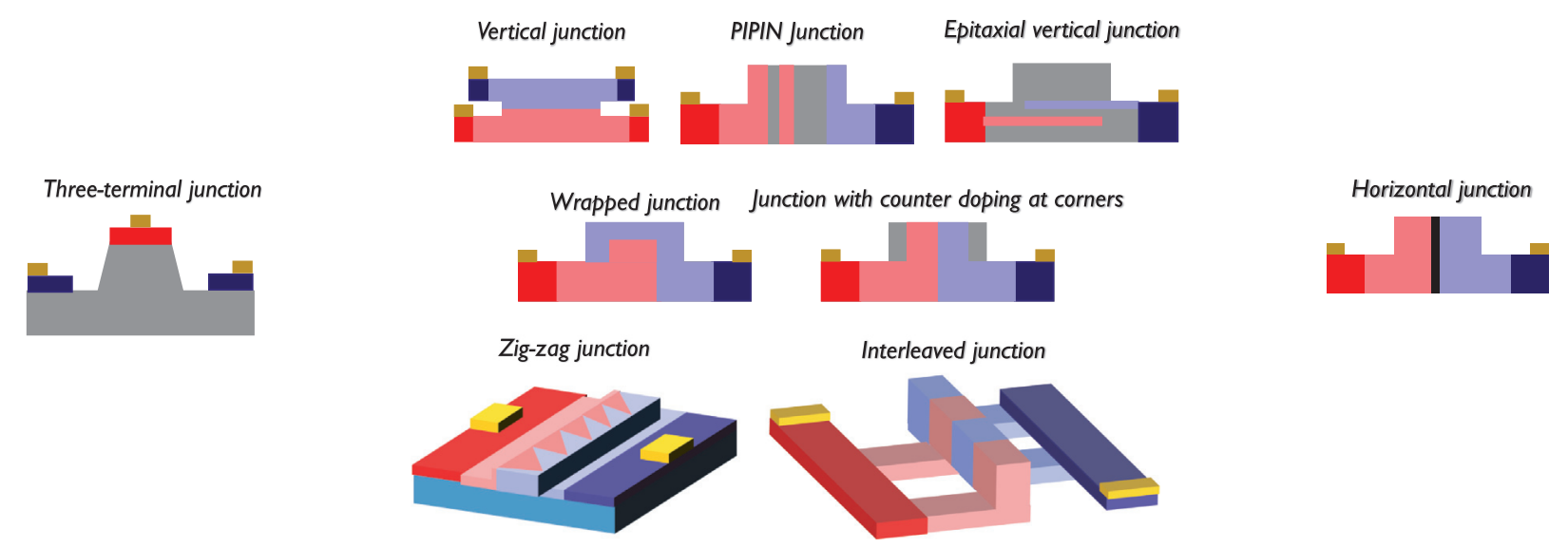

Various configurations of carrier injection (left), carrier depletion (center), and carrier accumulation phase shifters using plasma dispersion effect

(b)

Fig. 1 (a) Baseline architecture of carrier injection, carrier depletion, and carrier accumulation plasma dispersion phase shifters. (b) Various configurations of plasma dispersion phase shifters. 
been reported in Ref. 67, where a side-wall corrugated MZM with a bandwidth of $12.5 \mathrm{GHz}$ is operated at $25 \mathrm{~Gb} / \mathrm{s}$ using a finite impulse response filter for frequency compensation necessary for the broadband operation. ${ }^{67}$ The demonstrated modulator had a $250-\mu \mathrm{m}$-long phase shifter that exhibited a propagation loss of $5.3 \mathrm{~dB} / \mathrm{mm}$.

The carrier accumulation plasma dispersion phase shifter requires forward biasing of a metal-oxide-semiconductor (MOS) capacitor. $^{2,68,101,102}$ A typical implementation of an MOS (also known as silicon-insulator-silicon capacitor-SISCAP) modulator comprises a vertical slot waveguide with an overlapping stack of a sub- $\mu \mathrm{m}$-sized thick p-type poly-silicon layer on top of a sub- $\mu$ m-sized thick n-type c-silicon layer with a few $\mathrm{nm}$ (typically $<20 \mathrm{~nm}$ ) thick insulating gate oxide layer in the middle (i.e., p-Si/insulator/c-Si vertical slot waveguide) - see Fig. 1(a). ${ }^{69,101,102}$ Under accumulation conditions, in which a positive bias is applied to the p-type layer, the vertically confined optical mode overlaps strongly with the highly capacitive gate section. ${ }^{101,102}$ This enables $<1 \mathrm{~V} \cdot \mathrm{cm}$ modulation efficiency for SISCAP modulators ${ }^{37}$ with state-of-the-art values as low as 0.2 and $0.16 \mathrm{~V} \cdot \mathrm{cm} .{ }^{5,68,69}$ Owing to this higher modulation efficiency, typically $\leq 0.5$-mm-long phase shifters with lumped electrical driving are implemented in SISCAP modulators. ${ }^{65,67,69,69,103}$ The higher capacitance, which leads to higher modulation efficiency, caps the modulation speed of SISCAP modulators. ${ }^{2,104}$ The modulation efficiency and speed can be traded-off in SISCAP modulators by adopting the capacitance to a value that can provide either an efficient modulator (i.e., high capacitance) with low $V_{\pi} \cdot L$ or high-speed modulator (i.e., low capacitance) without any degradation of the loss parameter for the modulator (the increase or decrease in capacitance is independent of the doping level and is controlled by the thickness of gate oxide and the relative permitivity of the material used as the gate oxide). ${ }^{2,70,101,102,104}$ Typically, a vertical capacitor configuration is used in SISCAP modulators due to the ease of fabrication. ${ }^{5,68,69,69,70,104}$ It is straightforward to fabricate by the deposition of poly-Si. ${ }^{2,101}$ Techniques such as epitaxial lateral growth $^{101,102}$ and laser-induced epitaxial growth crystallize the poly-silicon layer to overcome the higher scattering loss due to defects in the lattice. ${ }^{2,227}$ Recently, SISCAP modulators using a c-Si/insulator/c-Si in a vertical slot configuration ${ }^{70}$ and in a horizontal slot configuration ${ }^{103}$ without requiring any additional processing steps have been reported [see Fig. 1(b)]. One of the first $40 \mathrm{~Gb} / \mathrm{s}$ Mach-Zehnder SISCAP modulators relied on a vertical capacitor configuration, and it delivered an ER of $9 \mathrm{~dB} .{ }^{68}$ The design consisted of a $400-\mu \mathrm{m}$-long MOS capacitor with a $6.5-\mathrm{dB} / \mathrm{mm}$ loss delivering a $V_{\pi} \cdot L$ of $0.2 \mathrm{~cm}$ for the O-band. The same MZM is driven by a CMOS driver to demonstrate its low-power operation for NRZ-OOK and for advanced modulation formats such as PAM-4 using a segmented driver. ${ }^{5}$ A recent demonstration reports high modulation efficiency of $0.16 \mathrm{~V} \cdot \mathrm{cm}$ for $25 \mathrm{~Gb} / \mathrm{s}$ operation using a $60-\mu \mathrm{m}-$ long phase shifter with a loss of $3.5 \mathrm{~dB} / \mathrm{mm}$. ${ }^{69}$ The MZM provided one of the best FoMs $\left(\alpha \cdot V_{\pi} \cdot L\right)$ of $<7 \mathrm{~dB} \cdot \mathrm{V}$. A microdisk-based SISCAP modulator demonstrated $15 \mathrm{~Gb} / \mathrm{s}$ operation on a small footprint of $\sim 30 \mu \mathrm{m}^{2}$ consuming only $55 \mathrm{fJ} / \mathrm{bit}^{70}$ In order to deliver $>50 \mathrm{~Gb} / \mathrm{s}$ operation using SISCAP modulators, a parametric study has been performed validating the trade-off between high-speed operation, modulation efficiency, and loss. ${ }^{104}$

Depletion of electrons and holes by reversely biasing a PN junction built in or around it is the most widely adopted scheme to implement the plasma dispersion-based phase modulator. ${ }^{8-11,13-19,22,32,33,71,71-87,106-121}$ Depletion-based modulators were the first all-silicon modulator to reach speeds of $40^{106}$ and $50 \mathrm{~Gb} / \mathrm{s}^{16}$ The current highest reported data rates of $100 \mathrm{~Gb} / \mathrm{s}$ using OOK modulation were also demonstrated in carrier depletion modulators. ${ }^{122,123}$ Carrier depletion phase shifters exhibit a relatively limited capacitance of 0.2 to $0.8 \mathrm{pF} / \mathrm{mm}^{21,91}$ and, hence, a limited modulation efficiency. In order to improve the modulation efficiency, the capacitance can be increased by either shrinking the mode size (determined by the waveguide geometry) or by reducing the width of the depletion region (higher transition capacitance). ${ }^{21}$ The latter requires higher doping concentrations, which leads to higher loss due to free carrier absorption. Over the years, a wide variety of PN junction designs are reported to solve this challenge of optimizing modulator speed, efficiency, and loss at the same time. ${ }^{13,15,17,18,22,71-76,78,107-109,116,117,119}$ Distinct categories of PN junction implementation are as follows:

(i) Vertical $\mathrm{P}(\mathrm{I}) \mathrm{N}$ junction, in which the $\mathrm{PN}$ junction is horizontally placed along the top and bottom surface of the waveguide $^{18,107-111}$ [see Fig. 1(b)]. These junctions are known to provide higher overlap between the optical mode and the depletion region to improve the modulation efficiency (higher capacitance) and reduced power consumption. ${ }^{18,107-111}$

(ii) Horizontal or lateral PN junction, in which the PN junction is vertically placed side-by-side in the center of the waveguide (laterally symmetric design) ${ }^{17,116,117,119}$ [see Fig. 1(b)]. The horizontal PN junction is abundantly used in various adapted forms to improve the $\alpha \cdot V_{\pi} \cdot L$ of the modulator. A few examples include (a) lateral PN junction with an offset from the center of the active region of the modulator, ${ }^{13,22,71-76}$ and (b) lateral junction formed by placing a p-doped slit in the center of the waveguide $^{78}$ while leaving the waveguide edges or corners un-doped. ${ }^{15,107}$ The rationale of (a) and (b) is to exploit the higher index change and lower loss provided by the dominantly p-doped active region. ${ }^{13,15,71-75,78,107}$

(iii) Interleaved or interdigitated PN junction, which comprises an alignment tolerant junction implemented by alternating the p-type and n-type doping regions along the length of the waveguide $^{81,83,84}$ [see Fig. 1(b)]. This type of junction is known to provide a good modulation efficiency by trading-off the speed and power efficiency for such a junction. ${ }^{32,80-84}$

One of the first $40 \mathrm{~Gb} / \mathrm{s}$ modulators relied on a vertical PIN junction and delivered a modulation efficiency of $4 \mathrm{~V} \cdot \mathrm{cm}$ and phase shifter loss of $18 \mathrm{~dB} / \mathrm{cm}^{106}$ Using a vertical P(I)N junction design, an athermal microdisk modulator with a high modulation efficiency of $250 \mathrm{pm} / \mathrm{V}$ consuming $0.9 \mathrm{fJ} / \mathrm{bit}$ while operating at $25 \mathrm{~Gb} / \mathrm{s}$ using NRZ-OOK signaling has been reported. ${ }^{18}$ At $25 \mathrm{~Gb} / \mathrm{s}$, the modulator had an IL of $\sim 1 \mathrm{~dB}$ and an ER of $6 \mathrm{~dB}$. One of the highest operating speeds of $60 \mathrm{~Gb} / \mathrm{s}$ with $3.8 \mathrm{~dB}$ ER comprised a symmetric PN junction. ${ }^{17}$ In this case, the modulator operated in a series of push-pull modes by connecting back-to-back the phase shifter PN junction embedded on both arms of the MZI. The series connection of the two phase shifters halves the net capacitance, and the push-pull scheme limits the chirp of the modulator. The modulator shows $50 \mathrm{GHz}$ bandwidth at $4 \mathrm{~V}$ reverse bias and an average modulation efficiency of $3.2 \mathrm{~V} \cdot \mathrm{cm}$ for a 4-mm-long PN junction. ${ }^{17}$ In another implementation, a symmetric carrier depletion MZM with sub-1 V drive signal operated at $20 \mathrm{~Gb} / \mathrm{s}$ and delivered a power consumption as low as $200 \mathrm{fJ} / \mathrm{bit}^{118}$ 
Yet another variant of the symmetric lateral PN junction used the doping compensating technique ${ }^{120}$ to demonstrate a $50-\mathrm{Gb} / \mathrm{s}$ MZM comprising a 3-mm-long phase shifter with a modulation efficiency of $1.85 \mathrm{~V} \cdot \mathrm{cm}$ and a loss of $3.9 \mathrm{~dB}$. An RM relying on a symmetric phase shifter configuration delivered a modulation efficiency of $25 \mathrm{pm} / \mathrm{V}$ at $0 \mathrm{~V}^{119}$ to demonstrate $36 \mathrm{fJ} / \mathrm{bit}$ energy consumption for $40 \mathrm{~Gb} / \mathrm{s}$ operation on a compact footprint of $0.5 \mathrm{~mm}^{2}$. A recent implementation demonstrates a 64-Gb/s MZM, in which the carrier depletion-based phase shifter is implemented through a $200-\mu \mathrm{m}$-long photonic crystal slow light structure. ${ }^{114}$ The modulator used a meandered RF electrode terminated with a $20 \Omega$ resistor to provide a bandwidth of $38 \mathrm{GHz}$ by preventing the velocity mismatch of the electrical and optical signals. The modulator required a voltage swing of $5.5 \mathrm{~V}$ to provide an energy consumption of $21 \mathrm{pJ} / \mathrm{bit}$ and provided $4.8 \mathrm{~dB}$ ER. The literature also reports various other demonstrations of asymmetric (where there is an offset of the PN junction from the waveguide center) carrier depletion-based phase shifters. ${ }^{8,16,72,74,76}$ For example, a Michelson interferometer with a partially shifted $\mathrm{PN}$-junction provided one of the highest modulation efficiencies of $0.72 \mathrm{~V} \cdot \mathrm{cm}$ at $1 \mathrm{~V}$ reverse bias and $4.7 \mathrm{~dB}$ loss for a $500-\mu \mathrm{m}$-long PN junction with a lumped driving scheme to operate at $40 \mathrm{~Gb} / \mathrm{s}$ through a bandwidth extension scheme involving a resistor parallel to the PN junction. ${ }^{8}$ The asymmetric PN diodes are also exploited by $\mathrm{RRs}^{72,76}$ to demonstrate low-power (sub-100 fJ/bit) and compact depletion modulators. The literature also reports fully shifted PN junctions, where a self-aligning process allows for the alignment of the n-doped region to the edge of the waveguide. ${ }^{16,74}$ In one implementation of a fully shifted PN junction, a 50-Gb/s MZM modulator provided a modulation efficiency of $2.8 \mathrm{~V} \cdot \mathrm{cm}$ and an IL of $4 \mathrm{~dB}$ for a 1-mm-long phase shifter. ${ }^{16}$ By further optimizing the implementation of self-aligned PN junctions, ${ }^{74} \mathrm{a}$ demonstration provided an $\sim 1 \mathrm{~dB} / \mathrm{mm}$ loss without compromising the speed of the modulator. The self-aligning process is further exploited to implement a PIPIN phase shifter with a modulation efficiency of $3.5 \mathrm{~V} \cdot \mathrm{cm}$ for a $0.95-\mathrm{mm}$-long phase shifter introducing $4.5 \mathrm{~dB}$ optical loss when operated at $40 \mathrm{~Gb} / \mathrm{s}^{79}$ Depletion-based modulators relying on an interleaved phase shifter design have also delivered high-speed operation. A modulator comprising a 3-mm-long interleaved phase shifter reported a high modulation efficiency of $0.62 \mathrm{~V} \cdot \mathrm{cm}$ with a static IL of $2.8 \mathrm{~dB}$ while delivering $40 \mathrm{~Gb} / \mathrm{s}$ modulation speed. ${ }^{81}$ An RM comprising a ring resonator with a $100-\mu \mathrm{m}$ radius, and an MZM with a $0.95-\mathrm{mm}$ phase shifter length operated at $40 \mathrm{~Gb} / \mathrm{s}$ speed using an interleaved phase shifter delivering a modulation efficiency and loss of $2.4 \mathrm{~V} \cdot \mathrm{cm}$ and $2.1 \mathrm{~dB} / \mathrm{mm}$, respectively. ${ }^{32}$ Apart from standalone implementations of highspeed phase shifters through these three baseline junction configurations and their adaptations, there are reports on junction implementations that rely on combining these three schemes in a single PN junction design. ${ }^{9,33,79,85,86}$ An example is the substrate-removed "zig-zag" phase shifter for an MZM delivering $1.6 \mathrm{~V} \cdot \mathrm{cm}$ modulation efficiency for a 2-mm-long phase shifter with $4.4 \mathrm{~dB}$ loss. ${ }^{14}$ The modulator provided $55 \mathrm{GHz}$ bandwidth and modulation speed of $90 \mathrm{~Gb} / \mathrm{s}^{14,87}$ Another example includes a phase shifter for $40 \mathrm{~Gb} / \mathrm{s}$ operation of a racetrack RM by combining horizontal, vertical, and interleaved approaches. This implementation delivered a modulation efficiency of $0.76 \mathrm{~V} \cdot \mathrm{cm}$ at $-1 \mathrm{~V}$ reverse bias and a loss of $3.5 \mathrm{~dB} / \mathrm{mm} .{ }^{33} \mathrm{~A}$ recent result demonstrated an RM with a record low modulation efficiency of $0.52 \mathrm{~V} \cdot \mathrm{cm}$ by employing a phase shifter comprising a combination of horizontal and vertical junctions by wrapping the $n$-doped region over the intrinsic and the p-doped region. ${ }^{9}$ The modulator showed $50 \mathrm{GHz}$ bandwidth and delivered $70 \mathrm{fJ} /$ bit energy consumption for $64 \mathrm{~Gb} / \mathrm{s}$ operation.

In the discussion presented above, we have provided a summary of the latest developments made for the improvement of plasma dispersion effect-based modulators. Figure 1(a) represents the three baseline plasma dispersion phase shifter architectures and their respective biasing configuration. Figure 1(b) gives an overview of a variety of other phase shifter configurations for carrier injection, carrier depletion, and carrier accumulation phase shifters.

Table 2 summarizes the typical performance for the injection, accumulation, and depletion-type plasma dispersion modulators. Table 2 also presents the best-reported results for the modulation efficiencies, losses, data rates, and energy consumption. There is an interplay of a vast pool of variables, such as junction type and its actuation mechanism, doping concentrations (typically ranging from $\sim 10^{17}$ to $\sim 10^{18} \mathrm{~cm}^{-3}$ ) and their positions,

Table 2 Typical and state-of-the-art performance matrix for the plasma dispersion high-speed phase modulators. The parentheses contain the best-reported result for a performance attribute. The matrix includes the results reported for O-band and C-band demonstrations.

\begin{tabular}{|c|c|c|c|c|c|}
\hline Principle & $\begin{array}{c}\text { Modulation } \\
\text { efficiency } \\
V_{\pi} \cdot L(\mathrm{~V} \cdot \mathrm{cm}) \\
\end{array}$ & Loss $(\mathrm{dB} / \mathrm{cm})$ & $\begin{array}{l}\text { Length }^{\mathrm{a}} \text { of } \\
\text { phase shifter } \\
(\mathrm{mm})\end{array}$ & $\begin{array}{c}\text { Data rate }{ }^{b} \\
(\mathrm{~Gb} / \mathrm{s})\end{array}$ & Energy/bit (fJ/bit) \\
\hline Carrier injection $^{c}$ & $<0.5\left(0.058^{8}\right)$ & $\sim 70\left(28^{7}\right)$ & $\geq 0.1$ to $<0.3$ & $<40\left(70^{7}\right)$ & $\sim 1000$ for MZMs and RMs $\left(0.1^{4,98}\right)$ \\
\hline Carrier accumulation $^{d}$ & $<0.3\left(0.16^{69}\right)$ & 50 to $80\left(\sim 35^{69}\right)$ & $\leq 0.5$ & $\sim 40\left(40^{5}\right)$ & >200 for MZMs, <200 $\left(3^{105}\right)$ for $\mathrm{SLMs}^{9}$ \\
\hline Carrier depletion ${ }^{\mathrm{e}}$ & $\sim 2\left(0.52^{9}\right)$ & 10 to $30\left(2.6^{10}\right)$ & $>1$ & $>40\left(100^{122,123}\right)$ & $\sim 200$ for MZMs $\left(32.4^{19}\right),<40$ for RMs $\left(0.9^{18}\right)$ \\
\hline
\end{tabular}

${ }^{\text {aT }}$ The phase shifter lengths are quoted for the non-resonant implementations.

${ }^{b}$ The rates for an OOK modulation scheme. The data rates for the carrier injection scheme are presented for approaches that do not require preemphasis of the modulating signal.

${ }^{\mathrm{C}}$ Typical performance for carrier-injection plasma dispersion phase modulators is based on Refs. 7, 8, 37, 61-67, and 88-100.

'Typical performance for the carrier-accumulation plasma dispersion modulators is based on Refs. 5, 12, 68, 69, 69, 70, and 101-105.

${ }^{\mathrm{e}}$ Typical performance for the carrier-depletion plasma dispersion modulators is based on Refs. 8-11, 13-19, 22, 32, 33, 71-87, and 106-123.

'Based on 1D-SiPh crystal.

${ }^{9}$ For photonic crystal cavity SLM. 
the thickness of the slab waveguide (typically ranging from $1 / 3$ to $1 / 4$ of the guiding silicon thickness), the thickness of the guiding silicon layer (typically $<1 \mu \mathrm{m}$ ), choice of the passive structure for the translation of phase modulation to amplitude modulation, and the design of the driving electrode (lumped, TW, and segmented), which can influence the performance of plasma dispersion modulators. In many cases, one performance parameter has to be delicately traded-off for another specification. This makes the design of a plasma dispersion modulator, which can meet all of the performance requirements concurrently, far from trivial. Operation of plasma dispersion modulators at speeds $>70 \mathrm{~Gb} / \mathrm{s}^{7,14,122}$ consuming tens of fJ/bit with high modulation efficiency enabling micrometer-sized footprints has been accomplished. Recent developments have resulted in a co-designed $\mathrm{SiPh}$ modulatordriver offering $100 \mathrm{~Gb} / \mathrm{s}$ while preserving a good modulation efficiency ${ }^{122}$ and without any customized fabrication process development. The plasma dispersion modulators deliver sufficient linearity and ER to handle advanced coherent modulation schemes, ${ }^{3,55,56,124-126}$ such as QAM, and amplitude modulation schemes, $9,17,29,52,66,115,123$ such as PAM. Furthermore, the plasma dispersion modulators have shown modest chirp-induced penalty for short- and long-haul links. ${ }^{3,26,30}$ The co-design and co-optimization of the driver-modulator design is the key to taking the performance of plasma dispersion modulators to a new level. ${ }^{122}$

\section{New Materials on Silicon for High-Speed Modulators}

Recent years have seen growing efforts to integrate materials with strong electro-optic effects on SiPh platforms. ${ }^{153-192,192-208,228}$ The integration of new materials provides routes for optically broadband and energy-efficient high-speed modulation with little or no current flow. ${ }^{176,181,184,188,202}$ Furthermore, in some cases, these new materials provide an excellent decoupling between amplitude and phase modulation, which is difficult to achieve with contemporary all-silicon plasma dispersion modulation schemes. ${ }^{174,178,184}$

Through the wafer-scale integration of these new material systems with SiPh platforms, one can achieve impressive modulator performance, while at the same time preserving the benefits provided by the mature $\mathrm{SiPh}$ technology. ${ }^{181}$ These new materials can be broadly categorized into the following:

- (silicon-)germanium,

- ferroelectrics,

- III-V semiconductors,

- 2D materials, and

- organic (electro-optic) materials.

Early results of high-speed modulators by integrating electrooptic materials with the SiPh platforms have shown a lot of promise. Many research teams are attempting to take their maturity and performance beyond the performance of plasma dispersion modulators. The sections below discuss the key modulator implementations by integrating these materials into $\mathrm{SiPh}$ platforms.

\section{1 (Silicon-)Germanium}

The FK effect and the QCS effect provide possible routes to implement compact, low-power, and high-speed electro-absorption modulators (EAMs) in $\mathrm{SiPh}^{31,143,144,146,149}$ Under the influence of an applied electric field, the band edge tilts to enhance the absorption coefficient for GeSi bulk material (FK effect) or $\mathrm{Ge} / \mathrm{SiGe}$ quantum well (QW) structures (QCS effect). ${ }^{31,38,145,146}$ These effects are intrinsically fast through their sub-ps response time. ${ }^{34,229}$ The QCS effect is stronger than the FK effect ${ }^{134,230}$ due to the strong excitonic effect and discretized density of states. The integration of bulk $\mathrm{GeSi}$ or $\mathrm{Ge} / \mathrm{SiGe} \mathrm{QW}$ requires epitaxial growth on SiPh platforms. The FK effect is considered more attractive than the QCS effect in the context of integration feasibility. The latter requires a more complex epitaxial growth process for band alignment and strain balancing. ${ }^{152,230}$ A large change in the absorption coefficient (typically ranging from 100 to $1000 \mathrm{~cm}^{-1}$ in the $\mathrm{C}$-band ${ }^{146}$ ) enables the implementation of a compact and energy-efficient EAM using the FK effect and QCS effect. ${ }^{31,230}$ Both effects rely on band engineering. Therefore, they have limited optical bandwidth $(\sim 30 \mathrm{~nm}$ for the FK effect and $\sim 20 \mathrm{~nm}$ for the QCS effect). ${ }^{134,149}$ In one of the first SiPh EAMs, the L-band absorption spectrum is shifted to the C-band by a tensiled $0.8 \%$ GeSi alloy. ${ }^{31}$ On a small footprint of only $30 \mu \mathrm{m}$, the EAM actuated by a vertical PIN diode provided an energy consumption of only $50 \mathrm{fJ} / \mathrm{bit}$. This EAM could operate over a 14-nm bandwidth from 1539 to $1553 \mathrm{~nm}$ and delivered an efficiency (defined as $\Delta \alpha / \alpha_{\mathrm{on}}$, where $\Delta \alpha$ is the absorption difference between ON-state and OFF-state, and $\alpha_{\text {on }}$ is the absorption for the ON-sate, in which no electric field is applied) of 2. Despite the limited bandwidth of $1 \mathrm{GHz}$, this promising demonstration triggered other demonstrations of EAMs. ${ }^{20,38,128,133-139,231,232}$ An evanescently coupled $55 \mu \mathrm{m}^{2} \mathrm{GeSi}$ EAM has been reported on a thick SOI platform (silicon guiding layer thickness of $3 \mu \mathrm{m}$ ), actuated by a horizontal PIN junction. ${ }^{135}$ This EAM is butt-coupled to the thick SOI waveguide through an appropriately designed taper. ${ }^{135}$ The modulator has an efficiency of $\sim 1$. This EAM operated in the C-band and provided $28 \mathrm{~Gb} / \mathrm{s}$ operation with $60 \mathrm{fJ} / \mathrm{bit}$ energy consumption. ${ }^{135}$ Literature also reports an L-band Ge EAM and a GeSi C-band EAM with an electrical 3-dB bandwidth of $\sim 50 \mathrm{GHz}$ for an optical bandwidth of $30 \mathrm{~nm} \cdot{ }^{136} \mathrm{In}$ this case, the EAM is butt-coupled to a silicon waveguide through a tapered waveguide section. The $\mathrm{Ge}$ or GeSi alloy, which is grown on a silicon recess, has a lateral PIN diode. A highly doped contact on the silicon layer biases the EAM. The modulator consumed $12.8 \mathrm{fJ} /$ bit energy at modulation speeds of $56 \mathrm{~Gb} / \mathrm{s}$ and provided an FoM of $<1$. Using this GeSi EAM, a digital to analog converter-less and digital signal processing (DSP)-free $100 \mathrm{~Gb} / \mathrm{s}$ transmission over a 500-m-long single-mode fiber using NRZ-OOK signaling has been reported. ${ }^{20,137}$

There are various reports on the implementation of the $\mathrm{Ge} /$ SiGe QCS effect-based modulators. ${ }^{141-152}$ In one case, a Ge/SiGe QW modulator is butt-coupled to a thin SOI rib waveguide in such a way that the QWs and the rib waveguide are vertically aligned with each other to have maximum overlap. ${ }^{147}$ The active section of the modulator, which comprised 15 pairs of $\mathrm{Ge} / \mathrm{Si}_{0.15} \mathrm{Ge}_{0.85}$ QWs, is driven by a vertical PIN diode. The $8-\mu \mathrm{m}^{2}$ modulator showed $3.5 \mathrm{GHz}$ bandwidth by using a $1-\mathrm{V}$ voltage swing (5.5 to $6.5 \mathrm{~V}$ ) to provide $\sim 3 \mathrm{~dB}$ of ER. In another implementation, Ge QWs are epitaxially grown at temperatures below $450^{\circ} \mathrm{C}$ on the silicon-rich $\mathrm{Si}_{1-x} \mathrm{Ge}_{x}$ low-loss waveguide platform. ${ }^{141}$ The $x$ concentration is selected such that it simultaneously ensures low indirect bandgap absorption and a sufficiently small lattice mismatch with the QWs. The $400-\mu \mathrm{m}^{2}$ modulator with $6.3 \mathrm{GHz}$ bandwidth and 0 to $3 \mathrm{~V}$ swing provided $\sim 4 \mathrm{~dB}$ ER and $3 \mathrm{~dB}$ IL. 
(Silicon-)Germanium Franz-Keldysh electro-absorption modulators in silicon photonics

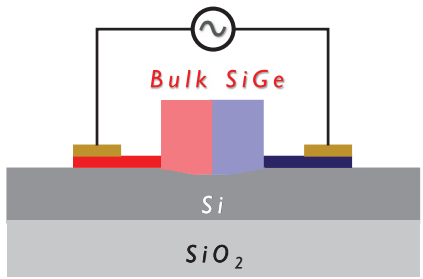

(Silicon-)Germanium EAMs are butt-coupled or evanescentcoupled with SiPh waveguides to demonstrate high-speed EAMs.

(a)
Quantum-confined Stark effect electro-absorption modulators in silicon photonics

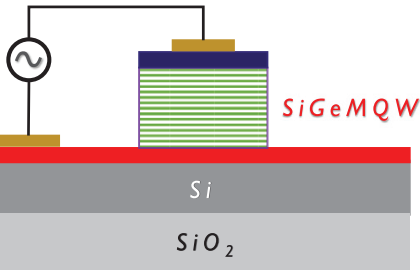

Germanium or silicon-germanium quantum wells are directly grown on SiPh waveguides or butt-coupled with SiPh waveguides

Demonstrated integration routes: monolithic

(b)

Fig. 2 Representative cross sections of (a) FK-based EAMs and (b) QCS effect-based EAMs in $\mathrm{SiPh}$. This figure also highlights the integration route for the respective EAMs. The term monolithic refers to the integration of a material with an SOI substrate using wafer-scale epitaxial growth resulting in PICs made out of one substrate technology in mass on a wafer level.

The FK effect or QCS effect electro-absorption amplitude modulators allow for the implementation of coherent $\mathrm{I} / \mathrm{Q}^{231,232}$ and advanced amplitude modulation schemes such as PAM-4. ${ }^{233}$ These modulation schemes are a promising solution for shortand long-reach links because they are spectrally efficient while being tolerant of optical fiber transmission impairments. ${ }^{54,55,231}$ The implementation of an EAM-based I/Q or PAM-4 modulators relies on balanced MZIs with an EAM and a fixed phaseshift in the arms of the MZI. ${ }^{231-233}$ Typically, an unequal splitting of the optical carrier is required by the splitter of the MZI to achieve the appropriate eye-opening for PAM-4 or constellation for the I/Q signal. ${ }^{233}$ Literature reports an EAM-based PAM-4 modulator by integrating an EAM in each arm of a two-port balanced MZI. ${ }^{231-233}$ In this modulator, a fixed phase shift is provided by embedding a thermal phase shifter in one of the two arms of the MZI. The device modulated a 128-Gb/s PAM-4 signal without requiring any DSP. Furthermore, there are reports on the demonstrations of modulating QPSK and 16-QAM signals using EAM-based coherent modulators. ${ }^{231,232}$ The energy consumption of these modulators takes a hit due to the powerhungry thermo-optic effect required to provide a fixed phase shift in the arms of the MZIs.
Figure 2 represents the typical cross sections of FK EAMs and QCS effect-based EAMs in SiPh. This figure also summarizes the integration routes of these materials with $\mathrm{SiPh}$. Table 3 summarizes the typical and the best-reported performance for the (silicon-)germanium modulators. This table covers the performance of the FK effect-based EAMs as well as QCS effect-based EAMs. The FoM for both FK and QCS effect-based EAMs is typically $<2$, although QCS effect-based modulators can potentially deliver better FoM due to their stronger excitonic absorption peaks. ${ }^{14,149,152}$ In most cases, FK-effect-based EAMs operate either in the L-band or C-band. ${ }^{150,152}$ It is possible to tune the operating wavelength of the QCS effect modulators by a bias voltage. ${ }^{150,152}$ Therefore, the QCS effect modulators can operate in the O-band as well. In terms of high-speed operation, FK effect-based EAMs have reached line rates of up to $100 \mathrm{~Gb} / \mathrm{s}$ for OOK modulation schemes, ${ }^{137}$ whereas the QCS effect-based modulators have not yet demonstrated such a high speed of operation.

\subsection{Ferroelectrics}

Ferroelectrics are materials possessing spontaneous polarization that can be altered by the application of a sufficiently strong

Table 3 Typical and state-of-the-art performance matrix for amplitude modulation by the FK effect and QCS effect. The best-reported performance attributes are mentioned in parentheses.

\begin{tabular}{lccccc} 
Principle & FoM ER/L & Loss $(\mathrm{dB})$ & $\begin{array}{c}\text { Modulator } \\
\text { length }(\mathrm{mm})\end{array}$ & Data rate $(\mathrm{Gb} / \mathrm{s})^{\mathrm{a}}$ & $\begin{array}{c}\text { Energy/bit } \\
(\mathrm{fJ} / \mathrm{bit})\end{array}$ \\
\hline FK effect $^{\mathrm{b}}$ & $<2\left(2^{31}\right)$ & $<6\left(4.8^{133}\right)$ & $\sim 0.050$ & $>40\left(100^{137}\right)$ & $<50\left(13^{20}\right)$ \\
Quantum-confined Stark effect $^{c}$ & $<2\left(7.9^{149}\right)$ & $<6\left(1.3^{149}\right)$ & $<0.25$ & $<10\left(7^{147}\right)$ & $<100\left(16^{148}\right)$ \\
\hline
\end{tabular}

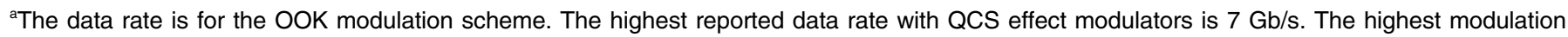
bandwidth has been reported to be $23 \mathrm{GHz}$.

${ }^{\text {b} T y p i c a l ~ p e r f o r m a n c e ~ f o r ~ t h e ~ F K ~ E A M s ~ i s ~ b a s e d ~ o n ~ R e f s . ~ 20, ~ 31, ~ 38, ~ 128, ~ a n d ~ 133-139 . ~}$

'Typical performance for the QCS EAMs is based on Refs. 141-152. 
electric field. ${ }^{234}$ As ferroelectric materials are non-centrosymmetric, they exhibit a Pockels effect. ${ }^{234}$ Many ferroelectrics have large Pockels coefficients compared to non-ferroelectric inorganic materials. The dominant contribution to the Pockels coefficients in these ferroelectrics tends to come from lattice vibrations rather than electron movements. ${ }^{234,235}$ Because of their strong Pockels effect, the integration of ferroelectric materials such as $\mathrm{LiNbO}_{3},{ }^{167-174}$ barium titanate $\left(\mathrm{BaTiO}_{3}\right),{ }^{175-182}$ and lead zirconate titanate (PZT) ${ }^{183-185}$ with $\mathrm{SiPh}$ provides a promising route for the implementation of efficient, high-speed modulators. ${ }^{2,175,228}$ A variety of integration techniques have been explored, such as molecular beam epitaxy (MBE), ${ }^{176,178}$ pulsed laser deposition, ${ }^{236,237}$ chemical solution deposition (CSD), ${ }^{238} \mathrm{RF}$ magnetron sputtering, ${ }^{239}$ metal organic chemical vapor deposition, ${ }^{240}$ and atomic layer deposition. ${ }^{241}$ The two key requirements for integrating ferroelectric materials with $\mathrm{SiPh}$ are to ensure a high electro-optic coefficient and low propagation loss. ${ }^{182}$ The following sections will review the latest developments to implement high-speed modulators by integrating ferroelectric materials with $\mathrm{SiPh}$.

\subsection{1 $\mathrm{LiNbO}_{3}$ on silicon (nitride) high-speed modulators}

$\mathrm{LiNbO}_{3}$ is a widely used material for modulators and provides high-speed devices with a bandwidth of $>35 \mathrm{GHz} \cdot{ }^{27,242,243}$ $\mathrm{LiNbO}_{3}$ modulators have been used for a long time in fiberoptic networks. ${ }^{242,244}$ These devices are based on low-indexcontrast waveguides in bulk $\mathrm{LiNbO}_{3},{ }^{242}$ in which the index contrast between the core and cladding of a $\mathrm{LiNbO}_{3}$ waveguide is provided by the diffusion of $\mathrm{Ti}$ or $\mathrm{Li}$ ions. ${ }^{242}$ The weak optical guiding resulting from the low-index-contrast requires the electrical contacts to be placed far away from the waveguides, resulting in typical $V_{\pi} \cdot L$ of 10 to $20 \mathrm{~V} \cdot \mathrm{cm}^{242}$ Recent demonstrations of high-index-contrast $\mathrm{LiNbO}_{3}$-on-insulator (LNOI) modulators have shown energy-efficient operation (a few tens of fJ/bit energy consumption) with 100-GHz bandwidth, $\sim 2 \mathrm{~V} \cdot \mathrm{cm}$ modulation efficiency, and $<0.5 \mathrm{~dB}$ loss for a device that is a few millimeters long (typically 2 to $20 \mathrm{~mm}$ long). ${ }^{243,245-247}$ These thin-film LNOI modulators rely on $\mathrm{LiNbO}_{3}$ layers of a few hundred nanometers thickness, which are bonded on top of $\mathrm{SiO}_{2}{ }^{243}$ Several approaches have been reported in the last two decades to integrate $\mathrm{LiNbO}_{3}$ with silicon at room temperature ${ }^{245,248,249}$ and to demonstrate high-speed modulation by bonding $\mathrm{LiNbO}_{3}$ with silicon ${ }^{167-169,250}$ or silicon nitride. ${ }^{170-172}$ The Pockels coefficient of integrated $\mathrm{LiNbO}_{3}$ is comparable to that of bulk $\mathrm{LiNbO}_{3},{ }^{249}$ but most of these integration attempts have resulted in modulator bandwidths that are smaller than the all-silicon modulator implementations. ${ }^{173}$ Recently, a tremendous boost in the speeds (not yet the overall performance) of such modulators has been reported. ${ }^{173,174}$ For example, for a 5-mm long TW-MZM that was realized by direct oxide bonding of thin-film $\mathrm{LiNbO}_{3}$ on top of a silicon waveguide, a bandwidth of $100 \mathrm{GHz}$ and a loss of $7.6 \mathrm{~dB}$ have been reported. ${ }^{173}$ The modulator had a limited efficiency of $6.7 \mathrm{~V} \cdot \mathrm{cm}$ due to $<100 \%$ vertical coupling of light into the unpatterned $\mathrm{LiNbO}_{3}$ membrane on top of the silicon waveguide. In another implementation, the complete coupling of light from a silicon waveguide to a patterned $\mathrm{LiNbO}_{3}$ rib waveguide through a vertical adiabatic coupler resulted in a modulation efficiency of $\sim 2.5 \mathrm{~V} \cdot \mathrm{cm} .{ }^{174}$ With a 2.5-dB loss, the 5-mm-long $\mathrm{LiNbO}_{3}$ phase shifter embedded in an MZI and driven by a TW electrode in the push-pull configuration provided $70 \mathrm{GHz}$ bandwidth and enabled single- lane $100 \mathrm{~Gb} / \mathrm{s}$ NRZ-OOK transmission. Unlike the other reported implementation, ${ }^{173}$ the integration of $\mathrm{LiNbO}_{3}$ with the SiPh PIC was carried out using BCB-bonding. Monolithic integration of $\mathrm{LiNbO}_{3}$ with SiPh PICs has yet to be reported.

\subsection{2 $\mathrm{BaTiO}_{3}$ on silicon high-speed modulators}

In recent years, there has been a lot of interest in the integration of $\mathrm{BaTiO}_{3}$ with $\mathrm{SiPh}^{175-182} \mathrm{BaTiO}_{3}$ is a promising material for integration with $\mathrm{SiPh}$ because of (a) its high Pockels coefficient [in bulk form, $\mathrm{LiNbO}_{3}$ has a Pockels coefficient of $\sim 30 \mathrm{pm} / \mathrm{V},{ }^{27,175,249}$ whereas BTO offers one of the highest Pockels coefficients $(>1000 \mathrm{pm} / \mathrm{V})$ in bulk form ${ }^{176,251}$ ]; (b) feasibility of integration with a silicon substrate, ${ }^{252}$ (c) capability to offer high-speed modulation; ${ }^{253}$ (d) high chemical and thermal stability of BTO; and (e) feasibility of low-loss hybrid BTO-Si passive circuitry. ${ }^{178,179}$ The integration of BTO with $\mathrm{SiPh}$ relies on epitaxial growth enabling integration compatible with 200 and $300 \mathrm{~mm}$ silicon wafers. ${ }^{181}$ The large lattice mismatch $(\sim 27 \%)$ between BTO and $\mathrm{Si}$ makes the integration of the two materials far from trivial. ${ }^{254} \mathrm{~A} \mathrm{SrTiO}_{3}$ (STO) buffer layer of a couple of nanometers thickness reduces this lattice mismatch to $<2 \%$. ${ }^{228}$ Among the various possible routes to grow BTO on SiPh platforms, MBE is known to provide the highest $(>900 \mathrm{pm} / \mathrm{V})$ Pockels coefficient in BTO thin films. ${ }^{176}$ In one approach, the BTO growth is done directly on an SOI wafer. ${ }^{177}$ Afterward, an amorphous silicon layer with a thickness equal to the thickness of the c-Si layer of the SOI wafer is grown. The resulting heterostructure is patterned lithographically to form a horizontal slot waveguide. Stronger guiding of the optical signal in the low-index BTO layer $\left(n_{\text {BTO }} \sim 2.38\right)$ enhances the overlap of the optical and electrical field in the BTO layer. This approach provided a Pockels coefficient of $213 \pm 49 \mathrm{pm} / \mathrm{V} .{ }^{177}$ A ring-based modulator with a modulation efficiency of $1.5 \mathrm{~V} \cdot \mathrm{cm}$ and a bandwidth of $4.9 \mathrm{GHz}$ has been reported, where the limited bandwidth is associated with the parasitic electrical effects. ${ }^{177}$ A key challenge faced by the integration approach mentioned in Ref. 177 and other integration approaches ${ }^{176,178}$ is the high propagation loss $(>40 \mathrm{~dB} / \mathrm{cm}) \cdot{ }^{176-178,255}$ The origin of this high loss is attributed to the absorption of hydrogen by the STO seed layer during the fabrication of the slot waveguide. ${ }^{178}$ Furthermore, it has been shown that a loss of $6 \mathrm{~dB} / \mathrm{cm}$ in a strip-loaded horizontal slot waveguide can be achieved by low-temperature annealing. ${ }^{178}$ Apart from the aforementioned approach, where BTO is grown directly on an SOI wafer, ${ }^{177}$ another possible approach entails the formation of a $\mathrm{BTO} / \mathrm{Si}$ heterostructure by bonding an SOI wafer with a BTO layer grown on a host SOI wafer. ${ }^{17,179}$ For such a bonded BTO/Si heterostructure, a Pockels coefficient of $923 \mathrm{pm} / \mathrm{V}$ has been reported (30 times larger than that of a $\mathrm{LiNbO}_{3}$ structure). ${ }^{179}$ In one implementation, a plasmonic MZM with a 10- $\mu \mathrm{m}$-long BTO/Si phase shifter with $25 \mathrm{~dB}$ loss delivered $72 \mathrm{~Gb} / \mathrm{s}$ and required a $V_{\pi}$ of $14.5 \mathrm{~V}$ (modulation efficiency of $0.0145 \mathrm{~V} \cdot \mathrm{cm}$ ). ${ }^{180}$ The modulator operated successfully for temperatures up to $130^{\circ} \mathrm{C}$. Furthermore, a wafer-level bonding of Si/BTO heterostructures on the back-end-of-line (BEOL) dielectric layer of an advanced SiPh platform has been reported. ${ }^{181}$ This integration led to the demonstration of an MZM with a limited bandwidth of $2 \mathrm{GHz}$ (mostly limited by the electrical bandwidth of the electrodes). ${ }^{181}$ The modulator had a modulation efficiency of $0.2 \mathrm{~V} \cdot \mathrm{cm}$ and loss of $<6 \mathrm{~dB}$ for a 1-mm-long phase shifter. ${ }^{181,182}$ 


\subsubsection{PZT on silicon nitride high-speed modulators}

PZT is an orthorhombic lead-containing ceramic ferroelectric material. ${ }^{256,257}$ Though BTO has a large Pockels coefficient, its use in $\mathrm{SiPh}$ requires complex and expensive integration technology. ${ }^{178,181,182,255}$ As compared to BTO, PZT has a smaller Pockels coefficient, but it can be deposited by a low-cost and simple CSD method. ${ }^{185,257}$ Ultra-thin lanthanide-based intermediate seed layers enable the deposition of high-quality thin (up to $200 \mathrm{~nm}$ ) PZT layers on silicon, ${ }^{257}$ with Pockels coefficients up to $150 \mathrm{pm} / \mathrm{V} .{ }^{228} \mathrm{PZT}$ thin films have been integrated with silicon nitride photonic circuits. An RM with a bandwidth of $33 \mathrm{GHz}$ and an MZM with a bandwidth of $27 \mathrm{GHz}$ have been demonstrated, ${ }^{184}$ with a modulation efficiency of $3.2 \mathrm{~V} \cdot \mathrm{cm}$ and a loss of $1 \mathrm{~dB} / \mathrm{cm}$. The PZT layer was deposited on a planarized silicon nitride PIC (i.e., the PIC consisted of silicon nitride strip waveguides with oxide bottom and side cladding, while the top cladding was air). The PZT thin film was poled by applying a voltage of 60 to $80 \mathrm{~V}$ for 1 h. $^{185}$

Figure 3 represents the typical cross sections of ferroelectric modulators in $\mathrm{SiPh}$. This figure also summarizes the integration routes of the respective materials with $\mathrm{SiPh}$. Table 4 presents a summary of the typical and the best-reported modulation efficiencies, losses, data rates, and energy consumption for the ferroelectric-based modulators in $\mathrm{SiPh}$. For all three types (i.e., $\mathrm{LiNbO}_{3}, \mathrm{BTO}$, and PZT), modulation speeds $\geq 40 \mathrm{~Gb} / \mathrm{s}$ have been demonstrated. $\mathrm{LiNbO}_{3}$-based modulators are outstanding in terms of low-loss operation and have delivered speeds of $\sim 100 \mathrm{~Gb} / \mathrm{s}$. In terms of modulation efficiency, BTObased modulators have shown a lot of promise with typical reported values of $<0.5 \mathrm{~V} \cdot \mathrm{cm}$.

\subsection{Organic Electro-Optic Materials}

Organic electro-optic materials are typically based on $\pi$-conjugated molecules with strong electron donor and acceptor groups at the ends of the $\pi$-conjugated structure. ${ }^{188,258}$ The delocalized electrons associated with this $\pi$-conjugated structure are confined by a strongly asymmetric potential well, giving rise to large Pockels coefficients. ${ }^{188,258}$ In contrast to the ferroelectrics discussed above, the Pockels effect in these organic electrooptic materials is mostly electronic in nature and can thus provide an ultra-fast response. ${ }^{188,190,228,258}$ Organic electro-optic materials can be processed at room temperature using inexpensive solution-based techniques, whereas the growth of inorganic Pockels materials typically requires high temperatures and/or the use of vacuum equipment. ${ }^{177,182,188,255,258}$

A combination of organic materials with $\mathrm{SiPh}$ devices has resulted in the so-called silicon-organic hybrid ( $\mathrm{SOH}$ ) class of modulators. ${ }^{186-188,197}$ The underlying organic materials in $\mathrm{SOH}$ modulators are designed to provide high electrooptic activity with experimentally demonstrated values of $n^{3} r>2000 \mathrm{pm} / \mathrm{V} .{ }^{190}$ This results in modulators with a lowloss, ${ }^{194}$ a low-chirp parameter of $\alpha=-0.02,{ }^{193}$ and a high modulation efficiency $\left(V_{\pi} \cdot L \approx 0.03 \mathrm{~V} \cdot \mathrm{cm}\right),{ }^{190}$ along with a
LiN $\mathrm{OO}_{3}$ phase modulator integrated with silicon photonics

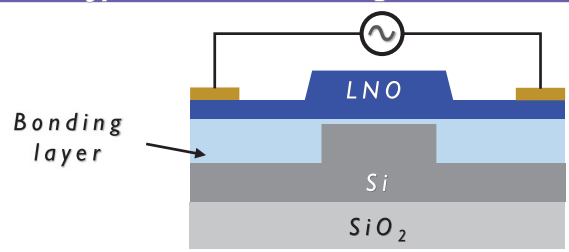

A thin film of LNO layer is bonded with SiPh platform. Patterning a rib waveguide in LNO enhances efficiency by virtue of 100\% coupling into LNO waveguide.

Demonstrated integration routes: bonding, transfer printing

(a)

PZT phase modulator integrated with silicon photonics

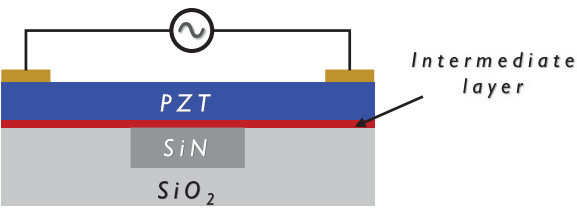

Chemical solution deposition of PZT on SiN waveguide by using ultra-thin lanthanide-based intermediate seed layer.

Demonstrated integration routes: deposition

(c)
BTO phase modulator integrated with silicon photonics

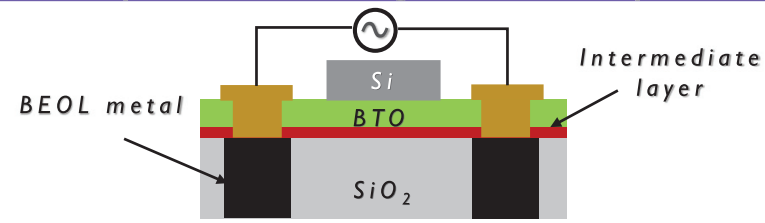

BTO grown on an unpatterned SOI wafer is transferred to the BEOL. of a SiPh process. The simpler approach of direct growth on patterned SOI have been futile so far. Demonstrated integration routes: monolithic, bonding

(b)

\section{Organics phase modulator integrated with silicon photonics}

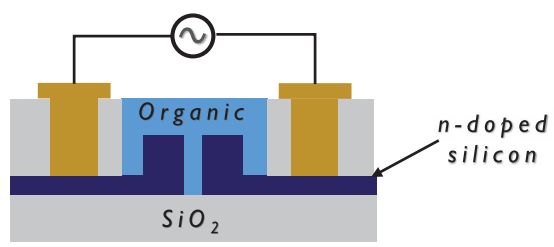

Highly electro-optic organic material is deposited on a processed slot waveguide after removing the BEOL stack.

Demonstrated integration routes: deposition

(d)

Fig. 3 Representative cross sections of (a) $\mathrm{LiNbO}_{3}$, (b) BTO, (c) PZT, and (d) organics modulators in SiPh. This figure also highlights the integration route for each material. The term monolithic refers to the integration of a material with a SiPh substrate using wafer-scale epitaxial growth resulting in PICs made out of one substrate technology in mass on a wafer level. 
Table 4 Typical and state-of-the-art performance matrix for the ferroelectric and organic high-speed phase modulators in SiPh. ${ }^{a}$ The parentheses contain the best-reported result for a performance attribute.

\begin{tabular}{|c|c|c|c|c|c|}
\hline Scheme & $\begin{array}{l}\text { Modulation efficiency } \\
\qquad V_{\pi} \cdot L(\mathrm{~V} \cdot \mathrm{cm})\end{array}$ & $\begin{array}{l}\text { Loss } \\
(\mathrm{dB} / \mathrm{cm})\end{array}$ & $\begin{array}{l}\text { Length of phase } \\
\text { shifter }(\mathrm{mm})\end{array}$ & $\begin{array}{l}\text { Data rate } \\
(\mathrm{Gb} / \mathrm{s})\end{array}$ & Energy/bit (fJ/bit) \\
\hline $\mathrm{LiNbO}_{3}$ integrated with $\mathrm{SiPh}^{\mathrm{c}}$ & $<3\left(2.2^{174}\right)$ & $\sim 1\left(0.98^{174}\right)$ & $>1$ & $>70\left(100^{174}\right)$ & $>100\left(170^{174}\right)$ \\
\hline $\mathrm{BaTiO}_{3}$ integrated with $\mathrm{SiPh}^{d}$ & $<0.5\left(0.2^{182}\right)$ & $>40\left(6^{182}\right)$ & $>1$ & $>40\left(72^{180}\right)$ & $<100^{179}$ \\
\hline PZT integrated with $\mathrm{SiPh}^{\mathrm{e}}$ & $1\left(1^{183}\right)$ & $1\left(1^{183}\right)$ & $>2$ & $40\left(40^{184}\right)$ & - \\
\hline Organics integrated with $\mathrm{SiPh}^{\dagger}$ & $<0.05\left(0.032^{190}\right)$ & $<45\left(20^{228}\right)$ & $<1.5$ & $>50\left(100^{196}\right)$ & $<100\left(0.7^{192}\right)$ \\
\hline
\end{tabular}

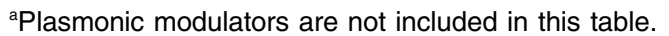

${ }^{\mathrm{b}}$ The data rates are reported for the OOK modulation scheme.

${ }^{\mathrm{C}}$ Typical performance for $\mathrm{LiNbO}_{3}$ integrated with SiPh is based on Refs. 167-174 and 228.

${ }^{d}$ Typical performance for $\mathrm{BaTiO}_{3}$ integrated with SiPh is based on Refs. 175-182 and 228.

eTypical performance for PZT integrated with SiPh is based on Refs. 183-185 and 228.

'Typical performance for the organics integrated with SiPh is based on Refs. 186-192, 192-200, and 228.

high modulation bandwidth $(>100 \mathrm{GHz})^{191,259}$ and low-energy consumption of a few fJ/bit. ${ }^{192,228}$ With $\pi$-voltages below $1 \mathrm{~V}$, SOH modulators may be directly driven by standard CMOS circuits without the need for any additional drive amplifiers, ${ }^{260}$ thereby opening a path toward greatly reduced power dissipation in highly integrated transceivers. For the fabrication of $\mathrm{SOH}$ devices, the organic electro-optic material is deposited onto fully processed $\mathrm{SiPh}$ chips in a postprocessing step, which requires removal of the BEOL layer stack of a standard $\mathrm{SiPh}$ process flow. ${ }^{228} \mathrm{SOH}$ modulators rely on slot waveguides, ${ }^{188,191}$ which guarantees enhanced interaction between the highly confined optical mode in the slot waveguide and the organic electrooptic material filling the slot. To pole the electro-optic material after deposition, the devices are heated, and a DC voltage is applied across the slot waveguides. This non-volatile poling transforms the random orientation of the electro-optic chromophores in the slot into an acentric orientation, thereby leading to a nonzero Pockels coefficient. After cooling down the device to ambient temperature, the voltage can be removed, and the poled electro-optic material preserves its orientation. $\mathrm{SOH}$ modulators have shown exemplary performance over the years. Some key results are reported in Refs. 189-191, 193, 196, and 261. The main skepticism faced by the $\mathrm{SOH}$ modulators comes from their long-term photochemical and photothermal stability. Recent research results reported in Refs. 195 and 262 have shown longterm stability of $\mathrm{SOH}$ devices following Telecordia standards. These early experiments have shown that in storing the $\mathrm{SOH}$ modulators at elevated temperatures of $85^{\circ} \mathrm{C}$, the degradation of the electro-optic activity converges to a stable level where the $V_{\pi} \cdot L$-product increases by $<15 \%$ after $2400 \mathrm{~h}$.

The limitations of poled organic materials can be overcome by substituting them with crystalline organic materials, which are inherently resilient to higher temperatures with good photochemical stability and do not require any poling. ${ }^{199}$ The electro-optic coefficients of the organic crystals are smaller (typically $\left.n^{3} r<500 \mathrm{pm} / \mathrm{V}\right)^{263}$ than those of the poled organic polymers. ${ }^{190}$ The integration of organic crystals requires growth to functionalize the silicon waveguides. ${ }^{198,199}$ An N-benzyl-2methyl-4-nitroaniline crystal has been deposited on an SOI waveguide to demonstrate an MZM that could operate at $12.5 \mathrm{~Gb} / \mathrm{s}$ with a modulation efficiency of $1.2 \mathrm{~V} \cdot \mathrm{cm}$ and a loss of $16 \mathrm{~dB}$ in the phase modulator with a $1.5 \mathrm{~mm}$ length. ${ }^{199}$

Typically, the analogue bandwidth of current $\mathrm{SOH}$ modulators is limited to $\sim 25 \mathrm{GHz}$ by the finite conductivity of the doped silicon slabs that provide an electrical contact with the rails of the slot waveguide. ${ }^{190}$ These bandwidths may be improved to more than $100 \mathrm{GHz}$ by exploiting optimized waveguide designs that rely on multi-step doping profiles. ${ }^{259}$ To that end, doped silicon slabs have also been replaced by BTO to feed the modulating RF signal to the EO material in the slot waveguide. ${ }^{200}$ This so-called capacitively coupled SOH MZM delivered a bandwidth of $65 \mathrm{GHz}$, which was sufficient to produce a $100-\mathrm{Gb} / \mathrm{s}$ OOK signal. ${ }^{200}$ The modulator had a modulation efficiency of $0.13 \mathrm{~V} \cdot \mathrm{cm}$; the loss from the phase shifter section is not reported. Yet another variant of the $\mathrm{SOH}$ modulator is the plasmonic-organic hybrid $(\mathrm{POH})$ modulator, ${ }^{258,264-269}$ where the silicon slot waveguide is replaced by a metal plasmonic slot waveguide to provide even higher optical confinement. ${ }^{258,265}$ The deposition of an organic electro-optic material on the metal slot waveguide leads to compact highspeed devices with bandwidths of hundreds of gigahertz. ${ }^{26,270}$ $\mathrm{POH}$ modulators are compact with typical lengths of a few microns and offer a low-energy consumption of a few tens of fJ/bit along with ultra-low voltage-length products of typically $\sim 0.005 \mathrm{~V} \cdot \mathrm{cm}^{258}$-nearly an order of magnitude lower than those of classical $\mathrm{SOH}$ modulators. The obvious limitation of the $\mathrm{POH}$ modulators is the high propagation loss in the plasmonic slot-waveguide section with typical values of $200 \mathrm{~dB} / \mathrm{mm} .{ }^{200,258}$ Despite the high efficiencies, this leads to substantial $\pi$-voltage-loss-products of the order of $10 \mathrm{~V} \cdot \mathrm{dB} .{ }^{188}$

Table 4 provides a summary of typical modulation efficiencies, losses, speeds, and energy consumption for organic electro-optic modulators in SiPh. When compared with the other Pockels modulators, the organic electro-optic modulators provide the best modulation efficiency with a reported value of $0.032 \mathrm{~V} \cdot \mathrm{cm} .{ }^{190}$ Organic electro-optic modulators have shown up to $100 \mathrm{~Gb} / \mathrm{s}^{196}$ operation and sub-fJ/bit energy consumption. ${ }^{192}$ Figure $3(\mathrm{~d})$ represents the typical cross section of organics modulators in $\mathrm{SiPh}$.

\subsection{III-V Semiconductors}

The integration of III-V semiconductors with $\mathrm{SiPh}$ platforms is a well-established research field, as it provides a viable route for the integration of laser sources with SiPh PICs. ${ }^{271-275}$ Direct bonding, ${ }^{272}$ BCB-aided adhesive bonding, ${ }^{271}$ III-V/Si flipchip bonding, ${ }^{275}$ and transfer printing ${ }^{274}$ are some of the most established bonding mechanisms for III-V/Si integration. 
Demonstrations of III-V integration with advanced SiPh platforms have been reported. ${ }^{276,277}$ Demonstrations of epitaxial growth of III-V on Si have also been reported despite the large lattice mismatch between $\mathrm{Si}$ and III-V (for example, InP has $8 \%$ lattice mismatch with $\mathrm{Si}) .^{278,279}$ The integration of III-V on silicon also provides a route for the implementation of efficient high-speed modulators because they provide (a) a large electron-induced refractive-index change, (b) a high electron mobility, and (c) a low carrier-plasma absorption. ${ }^{166}$

The reported implementations of III-V/Si modulators typically use a MOS (SISCAP) modulator architecture. ${ }^{161-163}$ In these modulators, n-doped III-V material, for example, InGaAsP, is bonded to a p-doped Si layer with an insulating layer separating the two. ${ }^{161-163}$ This results in a SISCAP architecture, where the insulating layer acts as a capacitor. A change in the refractive index takes place due to the charge accumulation due to the biasing of the doped silicon and III-V layer. According to Drude's model, the plasma dispersion induced refractive index change $-\Delta n$ is inversely proportional to the effective masses of the electrons and holes. ${ }^{61,89}$ As compared to the electron mass of $\mathrm{Si}$, the lighter electron mass of n-doped III-V results in a 17 times stronger electron-induced refractive index change. ${ }^{161}$ Furthermore, an $\mathrm{n}$-doped III-V provides a larger ratio of $-\Delta n$ to $\Delta k$, where $\Delta k$ is the change in the absorption coefficient, ${ }^{161}$ resulting in a phase modulator with reduced spurious intensity modulation ("pure-phase" modulation). Similarly, p-doped silicon provides larger $-\Delta n / \Delta k$ than p-doped III-Vs for carrier concentrations below $10^{19} \mathrm{~cm}^{-3} \cdot{ }^{161-163}$ Therefore, a hybrid III-V/Si MOS modulator brings the best of III-V and $\mathrm{Si}$ together. Consequently, this approach offers modulators with a 3 to 5 times better modulation efficiency compared to allsilicon MOS modulators and a phase shifter loss that is significantly lower than in all-silicon MOS modulators. ${ }^{161,163}$

In one report, an $\mathrm{InGaAsP} / \mathrm{Al}_{2} \mathrm{O}_{3} / \mathrm{Si}$ MOS structure provided a modulation efficiency of $0.047 \mathrm{~V} \cdot \mathrm{cm}$ and a phase shifter loss of $\sim 19.4 \mathrm{~dB} / \mathrm{cm}^{.161}$ In another report, an InGaAsP/ $\mathrm{SiO}_{2} / \mathrm{Si}$ MOS structure provided ${ }^{163}$ a modulation efficiency of $0.09 \mathrm{~V} \cdot \mathrm{cm}$ with an absorption loss of $26 \mathrm{~dB} / \mathrm{cm}$ in the phase shifter. Both demonstrations show an impressive modulation efficiency. But, the electro-optic cut-off frequency is rather limited due to the large oxide capacitance in the carrier accumulation mode of operation. ${ }^{161,163}$

One possible approach to break the trade-off between the high-speed operation and high modulation efficiency banks on operating the III-V on $\mathrm{Si}$ MOS phase shifter in reverse bias. ${ }^{165,280}$ The III-V on Si MOS modulator that is operated in reverse bias is architecturally similar to the forward biased III-V on Si modulator. ${ }^{161,163,165,280}$ For a given thickness of the oxide layer, the reverse biased III-V on Si phase shifter has a reduced depletion capacitance as compared to the accumulation capacitance. ${ }^{165,280}$ The combined plasma dispersion effect and FK effect in the reverse biased III-V on Si MOS phase shifter ensure a modulation efficiency that is comparable to that in the accumulation mode while ensuring a low-loss operation due to the limited hole density in the n-doped InGaAsP layer of the modulator. ${ }^{165,280}$ A III-V on Si MOS modulator with a 250- $\mu \mathrm{m}$-long phase shifter and a 9-nm-thick oxide layer has been reported with a modulation efficiency of $0.11 \mathrm{~V} \cdot \mathrm{cm} .{ }^{165}$ As compared to the carrier accumulation MOS, the reverse biased MOS provides a $3.3 \times$ lower depletion capacitance, hence paving the way for a $3.3 \times$ improvement in modulation bandwidth and energy consumption per bit. The implementation of III-V on $\mathrm{Si}$ MOS modulators is not limited to MZIs only. ${ }^{164}$ By implementing a III-V on Si MOS modulator in a racetrack configuration (implementing III-V on Si MOS modulators in a ring is challenging due to the long taper length needed for the III-V on Si hybrid waveguide), the energy consumption of a $40-\mathrm{GHz}$ (3-dB bandwidth) modulator has been improved by a factor of 3.7 as compared to the III-V on Si MZMs, while delivering a modulation efficiency of $0.064 \mathrm{~V} \cdot \mathrm{cm} .^{164}$ The $100-\mu \mathrm{m}$-long phase shifter section of the modulators comprised a thin aluminum oxide gate layer separating the n-type III-V and the p-doped Si layer. Recently, a proof-of-concept, taper-less integration of a few tens of nanometers thick III-V layer with $\mathrm{Si}$ has been demonstrated to reduce the footprint and loss of the device ${ }^{281}$ without compromising the modulation efficiency of the III-V on Si MOS capacitor.

Apart from phase modulators, EAMs have been reported by the bonding of III-V QWs with silicon. ${ }^{140,282}$ These EAMs use a PIN architecture, where the application of a bias voltage changes the optical absorption of the intrinsic multiple QWs due to the QCS effect. ${ }^{282} \mathrm{~A}$ segmented electrode, comprising a low-impedance active modulation section cascaded by a high-impedance passive section, provides high-bandwidth operation while ensuring low reflection and good modulation efficiency. ${ }^{140} \mathrm{~A}>67 \mathrm{GHz}$ bandwidth hybrid III-V on silicon EAM has been demonstrated using this scheme. ${ }^{140}$ In the O-band, this modulator has an IL of $4.9 \mathrm{~dB}$, and it shows $>9 \mathrm{~dB}$ of dynamic ER for $50 \mathrm{~Gb} / \mathrm{s}$ operation over a $16-\mathrm{km}$ long optical fiber link.

Figure 4 represents the typical cross sections of III-V on $\mathrm{Si}$ phase modulators and EAMs. This figure also summarizes the routes to integrate III-V on Si. Table 5 presents a summary of the typical and best-reported values for the modulation efficiencies, losses, data rates, and energy consumption for forward and reverse biased III-V on Si phase modulators as well as for III-V on Si EAMs. III-V on Si modulators have shown an impressive modulation efficiency and low-loss operation, but so far experimental demonstrations of high-speed operation $(\sim 100 \mathrm{~Gb} / \mathrm{s})$ remain elusive.

\subsection{D Materials}

Atomically thick 2D materials, the most prominent graphene, have received considerable interest in the last decade to implement efficient modulators by integration with $\mathrm{Si} / \mathrm{SiN}$ PICs. ${ }^{1,153-160,201-208}$ The implementation of graphene-based modulators in $\mathrm{SiPh}$ involves the transfer of the graphene layer on a Si or SiN waveguide. ${ }^{1}$ Mature and wafer-scale chemical vapor deposition is the widely adopted scheme to deposit graphene on a variety of metallic and dielectric substrates. ${ }^{283-285}$ The transfer of the graphene layer on a $\mathrm{Si}$ or $\mathrm{SiN}$ waveguide is performed after delaminating graphene from the substrate while adding a polymer carrier to handle the graphene film. ${ }^{154,159}$ Recently, microtransfer printing technology has shown promising results to transfer graphene to the target substrate in an automatic fashion. ${ }^{159}$ A single graphene layer, which is only a fraction of a nanometer thick, can absorb $2.3 \%$ of light ${ }^{286}$ with a wavelength ranging from the visible to $\mathrm{THz}$ wavelengths (graphene is a gapless material). Furthermore, graphene has a very high electron mobility of $>100,000 \mathrm{~cm}^{2} \cdot \mathrm{V}^{-1} \cdot \mathrm{s}^{-1}$ (silicon has a carrier mobility of $1400 \mathrm{~cm}^{2} \cdot \mathrm{V}^{-1} \cdot \mathrm{s}^{-1}$ ). ${ }^{228,287,288}$ These two properties enable the implementation of high-speed electro-absorptive ${ }^{153}$ or 
III-V on silicon phase modulators in silicon photonics (reverse biased and forwarded biased)

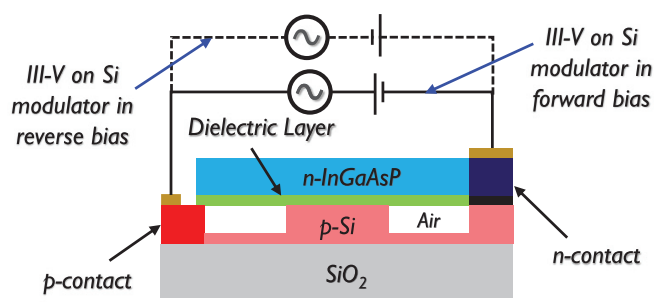

n-doped III-V material, such as InGaAsP, and p-doped silicon bonded with a few $\mathrm{nm}$ thick insulating layer separating the two materials to form III-V/Si MOS modulator, which can be operated either in forward bias or reverse bias.
III-V on silicon electro-absorption modulators in silicon photonics
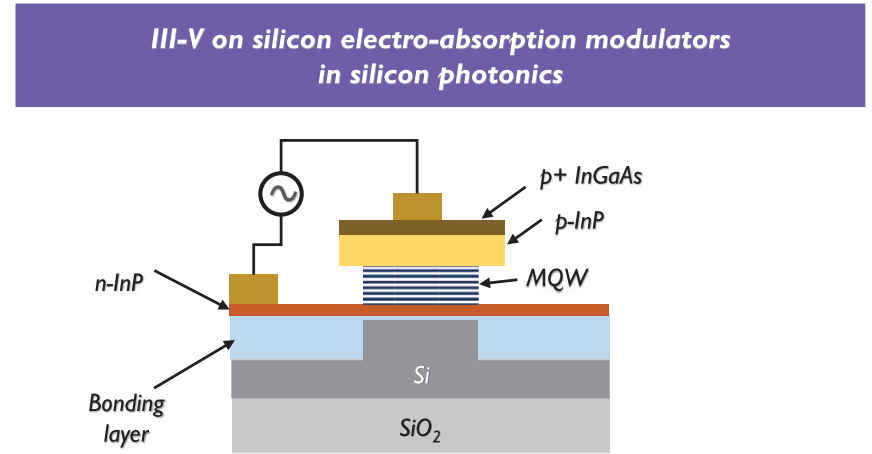

III-V on Si electro-absorption modulators by evanescent coupling of III-V bonded with silicon

Demonstrated integration routes: bonding, transfer printing

(b)

Fig. 4 Representative cross sections of (a) III-V on Si phase modulator and (b) III-V on Si EAM.

Table 5 Typical and state-of-the-art performance matrix for the III-V on Si high-speed modulators in SiPh. The parentheses contain the best-reported result for a performance attribute.

\begin{tabular}{|c|c|c|c|c|c|}
\hline Operation & $\begin{array}{l}\text { Modulation efficiency } \\
\qquad V_{\pi} \cdot L(\mathrm{~V} \cdot \mathrm{cm})\end{array}$ & $\begin{array}{c}\text { Loss } \\
(\mathrm{dB} / \mathrm{cm})\end{array}$ & $\begin{array}{l}\text { Length of phase } \\
\text { shifter }(\mathrm{mm})\end{array}$ & $\begin{array}{l}\text { Data rate }{ }^{\mathrm{a}} \\
(\mathrm{Gb} / \mathrm{s})\end{array}$ & $\begin{array}{c}\text { Energy/bit } \\
\text { (fJ/bit) }\end{array}$ \\
\hline $\begin{array}{l}\text { Forward biased III-V on } \mathrm{Si} \text { as a phase } \\
\text { modulator }^{\mathrm{d}}\end{array}$ & $<0.1\left(0.047^{161}\right)$ & $<30\left(19.4^{161}\right)$ & $<0.5$ & $>30\left(32^{163, b}\right)$ & $\geq 100$ \\
\hline $\begin{array}{l}\text { Reverse biased III-V on Si as a phase } \\
\text { modulator }^{d}\end{array}$ & $<0.2\left(0.11^{164}\right)$ & $\left(28^{280}\right)$ & $<0.5$ & $200^{c, 280}$ & $\leq 100^{280}$ \\
\hline Operation & FoM ER/IL & Loss (dB) & $\begin{array}{l}\text { Modulator length } \\
\qquad(\mathrm{mm})\end{array}$ & $\begin{array}{l}\text { Data rate }{ }^{a} \\
(G b / s)\end{array}$ & $\begin{array}{l}\text { Energy/bit } \\
\text { (fJ/bit) }\end{array}$ \\
\hline III-V on $\mathrm{Si}$ as an amplitude modulator ${ }^{\mathrm{d}}$ & $\sim 2\left(2^{140}\right)$ & $\left(4.9^{140}\right)$ & $\leq 0.1$ & $\sim 50(50)^{140}$ & - \\
\hline
\end{tabular}

${ }^{\mathrm{a}}$ The data rates are reported for the OOK modulation scheme.

${ }^{\mathrm{b}}$ The value represents the highest reported 3-dB bandwidth of $40 \mathrm{GHz}$ for a racetrack resonator modulator in III-V on $\mathrm{Si}$.

${ }^{\mathrm{c}}$ The value represents the theoretically predicted value for the 3-dB bandwidth in $\mathrm{GHz}$.

${ }^{\mathrm{d}}$ The typical performance of III-V on Si high-speed modulators is based on Refs. 140, 161-166, and 282.

electro-refractive ${ }^{201}$ modulators through the integration of graphene with SiPh ICs.

The two prominent types of graphene modulators in $\mathrm{SiPh}$ comprise a SiPh rib waveguide, which is either loaded with a single layer or a double layer of graphene ${ }^{153,156}$ (see Fig. 5). The operating principle of these modulators relies on the charging and discharging of a capacitor. A single-layer graphene modulator comprises a graphene loaded Si rib waveguide with a doped slab region. ${ }^{153} \mathrm{~A}$ few $\mathrm{nm}$ thick dielectric spacer, such as $\mathrm{SiO}_{2}, \mathrm{Al}_{2} \mathrm{O}_{3}$, or hBN, separates the waveguide and the graphene layer to form a capacitor. ${ }^{1}$ The first reported graphene modulator ${ }^{153}$ had a 4-dB ER and relied on a single-layer approach. The $25-\mu \mathrm{m}^{2}$ EAM provided $1 \mathrm{GHz}$ operation, limited by the RC time constant, and an electroabsorption modulation of $0.1 \mathrm{~dB} / \mu \mathrm{m} .{ }^{153}$ The absorption in graphene is dependent on the bias voltage. ${ }^{1}$ This voltage tunes the Fermi level of graphene to enable or inhibit the interband transitions by excitation of electrons through the incident photons. ${ }^{201,289}$ This change in absorption due to carrier accumulation in the graphene sheet results in modulation of light propagating in the waveguide. The doped $\mathrm{Si}$ waveguide in the single-layer graphene modulator is a source of loss, and it requires an implantation step. ${ }^{153,156}$ Double-layer graphene modulators remedy these limitations. ${ }^{156}$ They are separated by a dielectric layer that resides on top of a completely passive waveguide. (This architecture does not require any doping of the waveguide slab. ${ }^{156}$ The first doublelayer graphene modulator reported $1 \mathrm{GHz}$ bandwidth and an electroabsorption modulation of $0.16 \mathrm{~dB} / \mu \mathrm{m} .{ }^{156}$ The $40-\mu \mathrm{m}$ long modulator provided a $6-\mathrm{dB}$ ER and required a $5-\mathrm{V}$ driving voltage. One of the first multi-Gb/s graphene-silicon modulators ${ }^{157}$ relied on a single-layer graphene architecture that provided a 5.2-dB ER for a 50- $\mu$ m-long EAM operating at $\sim 2.5 \mathrm{~V}$. At $10 \mathrm{~Gb} / \mathrm{s}$, the modulator provided a modulation efficiency of $1.5 \mathrm{~dB} / \mathrm{V}$. The $\mathrm{ON}$ state loss is $3.8 \mathrm{~dB}$ with a power consumption of about $350 \mathrm{fJ} / \mathrm{bit}$. Another result reports a double-layer graphene $\mathrm{RM}^{158}$ using a critical coupling effect in a 40- $\mu \mathrm{m}$ SiN ring. In this implementation, a 50-nm thick $\mathrm{Al}_{2} \mathrm{O}_{3}$ layer separates the two graphene layers. By biasing, the change 


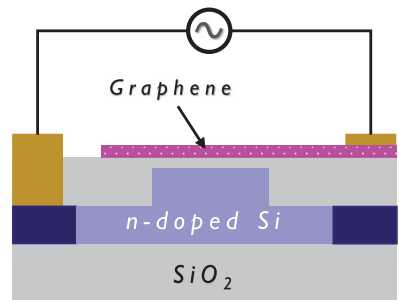

(a)

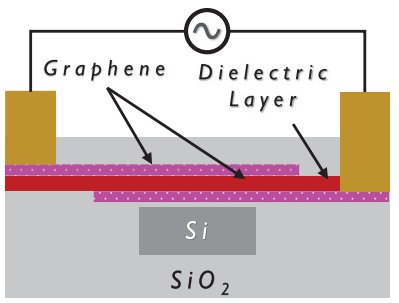

(b)
The implementation of graphene-based modulators in SiPh involves transferring a single layer of graphene or double layer of graphene on a Si or SiN waveguide to form a modulator.

Fig. 5 Representative cross sections of (a) single-layer graphene phase/amplitude modulator and (b) double-layer graphene amplitude/phase modulator in SiPh.

in the transparency of the graphene capacitor led to the modulation of the optical signal. The reduced capacitance resulting from the thick spacer between the graphene layers led to a modulator showing $30 \mathrm{GHz}$ bandwidth with a modulation efficiency of $1.5 \mathrm{~dB} / \mathrm{V}$ but required a $-30-\mathrm{V} \mathrm{DC}$ bias and

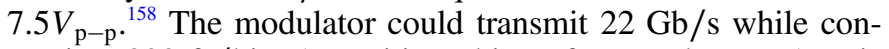
suming $800 \mathrm{fJ} /$ bit. A positive chirp of a graphene EAM is an interesting property. ${ }^{160}$ This feature is useful in compensating for the chromatic dispersion in an optical transmission link for the transmission of $10-\mathrm{Gb} / \mathrm{s}$ without any optical signal to noise ratio penalty. ${ }^{160}$

Apart from amplitude modulation, it is also feasible to use graphene for phase modulation. ${ }^{201-208}$ Phase modulation is more exciting for the encoding of complex modulation formats such as QPSK. A single-layer graphene architecture, in which the graphene layer is separated from the silicon waveguide by a thin dielectric spacer layer, acts as a phase modulator by shifting the Fermi level of graphene. ${ }^{201,205,207,290,291}$ A single-layer graphene phase modulator of $300 \mu \mathrm{m}$ length is integrated on a Si MZI and showed a modulation efficiency of $0.28 \mathrm{~V} \cdot \mathrm{cm}$. The modulator delivered an ER of $35 \mathrm{~dB}$, an $\mathrm{EO}$ bandwidth of $5 \mathrm{GHz}$, and a loss of $236 \mathrm{~dB} / \mathrm{cm}^{201}$ With a $2-V_{\mathrm{p}-\mathrm{p}}$ drive, the transmission of $10 \mathrm{~Gb} / \mathrm{s}$ over a $50-\mathrm{km}$ fiber span is demonstrated. The higher loss in the modulator is associated with the suboptimal transfer of graphene. ${ }^{201}$ The quality of graphene, which is typically determined by the scattering time and is related to the carrier mobility, plays a detrimental role in the performance of graphene modulators. ${ }^{1}$ Typically, scattering time of $>100 \mathrm{fs}$ is required to implement energy-efficient graphene modulators with a high ER. ${ }^{1}$ Achieving $10,000 \mathrm{~cm}^{2} \cdot \mathrm{V}^{-1} \cdot \mathrm{s}^{-1}$ graphene carrier mobility, which roughly corresponds to 300 fs scattering time, upon the complete modulator integration process would be ideal. Accomplishing this would lead to extremely low IL. ${ }^{289}$ In perspective, the combination of low IL in a dual-layer graphene structure, the high graphene electro-absorption, or electro-refractive efficiency would lead to a modulator with a good FoM. ${ }^{1}$ It is important to mention that graphene can be used for detection, ${ }^{1}$ and this makes graphene suitable for a telecommunication platform on a passive integrated circuit operating in several telecommunications bands.

Inspired by the promising results shown by graphene, other $2 \mathrm{D}$ materials such as tungsten disulfide $\left(\mathrm{WS}_{2}\right)$ and molybdenum disulfide $\left(\mathrm{MoS}_{2}\right)$ have also been integrated with $\mathrm{SiPh}$. Results show phase change relative to the change in absorption for these materials at telecom wavelengths when integrated with $\mathrm{SiN} .{ }^{204}$

Figure 5 represents typical cross sections of a single layer graphene modulator (a) and a double layer graphene modulator (b) in $\mathrm{SiPh}$. Table 6 provides the current state of high-speed phase and amplitude modulators in $\mathrm{SiPh}$ by integrating $2 \mathrm{D}$ materials. The currently reported performances of $2 \mathrm{D}$ modulators, in particular graphene modulators, are mostly limited by technological immaturity and not by conceptual limits. Graphene requires more development to reach the best performances. More specifically, graphene needs to preserve the high carrier mobility after the integration in the photonic circuit. This preservation is not the case yet. By achieving a high level of technological maturity, graphene has the potential to provide devices that can work over a wide range of wavelengths due to its gapless band structure, miniaturized devices due to its large EO efficiency, feasibility for BEOL integration with the $\mathrm{SiPh}$ fabrication process, and a possibility to offer a complete platform for communication applications, as graphene possesses properties for efficient modulation and detection.

\section{Summary and Outlook}

Years of academic and industrial R\&D have enabled a tremendous boost in the performance of plasma dispersion phase modulators. Operation at speeds of $100 \mathrm{~Gb} / \mathrm{s}$ with high modulation efficiency is now feasible. Plasma dispersion modulators can operate with a sub-1-V drive signal consuming a few tens of $\mathrm{fJ} / \mathrm{bit}$ in a $\mu \mathrm{m}$-sized footprint. Demonstrations of advanced modulation schemes such as PAM-4, PAM-8, QPSK, 16-QAM, and 32-QAM have been reported in plasma dispersion modulators. The modulators have an insignificant chirp-induced penalty for long- and short-haul links. A closer synergy through co-design and co-optimization of electronic-photonic modulator design is the key to take plasma dispersion modulators beyond $100 \mathrm{~Gb} / \mathrm{s}$ operation in an efficient manner. The last five years have seen a new paradigm in achieving higher speed and lower loss operations of $\mathrm{SiPh}$ modulators by integrating materials such as ferroelectrics, organics, SiGe alloys, III-Vs, and 2D materials. Unlike plasma dispersion modulators, which are commercially deployed and have a high manufacturing readiness level (MRL) of $>8$, these new modulators are still in the early 
Table 6 Typical and state-of-the-art performance matrix for graphene on Si high-speed modulators in SiPh. The parentheses contain the best-reported result for a performance attribute.

\begin{tabular}{|c|c|c|c|c|c|}
\hline Operation & $\begin{array}{l}\text { Modulation efficiency } \\
\qquad V_{\pi} \cdot L(\mathrm{~V} \cdot \mathrm{cm})\end{array}$ & Loss $(\mathrm{dB} / \mathrm{cm})$ & $\begin{array}{l}\text { Length of phase } \\
\text { shifter }(\mathrm{mm})\end{array}$ & $\begin{array}{c}\text { Data rate } \\
(\mathrm{Gb} / \mathrm{s})\end{array}$ & Energy/bit (fJ/bit) \\
\hline $\begin{array}{l}\text { 2D Materials on } \mathrm{Si} \text { as } \\
\text { phase modulator }\end{array}$ & $<0.5\left(0.28^{201}\right)$ & $>200\left(236^{201}\right)$ & $<0.5^{d}$ & $\sim 10\left(10^{201}\right)$ & $\begin{array}{l}\left.\sim 1000(600)^{1}\right) \text { for MZM; } \\
<500 \text { for RM }\end{array}$ \\
\hline Operation & FoM ER/IL & Loss (dB) & $\begin{array}{l}\text { Modulator Length } \\
\qquad(\mathrm{mm})\end{array}$ & Data rate $(\mathrm{Gb} / \mathrm{s})$ & Energy/bit (fJ/bit) \\
\hline $\begin{array}{l}\text { 2D Materials on } \mathrm{Si} \text { as } \\
\text { amplitude modulator }^{\mathrm{c}}\end{array}$ & $<3\left(4.9^{155}\right)$ & $<4\left(0.9^{203}\right)$ & $<0.15$ & $>10\left(50^{154}\right)$ & $\sim 100\left(40^{202}\right)$ \\
\hline
\end{tabular}

R\&D stage (MRL <7) and are currently not included in any commercial product.

Electro-absorption modulation by (silicon-)germanium FK or QCS devices allows for micrometer-sized footprints. FK-EA modulation provides $100 \mathrm{~Gb} / \mathrm{s}$ operation, but these modulators have a limited ER as well as limited operating optical bandwidth. QCS-EA modulation has integration challenges with $\mathrm{SiPh}$. Moreover, their operating speed is $<10 \mathrm{~Gb} / \mathrm{s} . \mathrm{LiNbO}_{3}$, $\mathrm{BaTiO}_{3}, \mathrm{PZT}$, graphene, and organic thin films on Si PICs offer high Pockels coefficients, paving the way for linear and chirpfree high-performance SiPh modulators. Early results reported in the literature indicate that promising performance is achievable by integrating thin-film $\mathrm{LiNbO}_{3}, \mathrm{BaTiO}_{3}$, or PZT materials on $\mathrm{Si}$. The integration of organic materials on $\mathrm{Si}$ has also shown $100 \mathrm{~Gb} / \mathrm{s}$ operation and impressive modulation efficiency. Progress has been reported to address the lifetime issues, but more development is necessary before the commercial launch of high-speed modulators relying on organic electro-optic materials. Bringing together III-V and Si brings the best out of III-V and Si to implement MOS-type phase modulators and EAMs. The product of $V_{\pi}$ and phase shifter loss is impressive for the III-V on Si phase modulators, but high-speed operation remains elusive. (Theoretically, they have shown impressive results.) Integrating 2D materials, in particular graphene, with $\mathrm{SiPh}$ has the potential to provide a complete solution for modulation and detection at high speed. Multi-Gb/s operation has been reported for the graphene phase and amplitude modulators. A technique to preserve the high carrier mobility after integration in the photonic circuit is essential to unleash the true potential of graphene modulators in $\mathrm{SiPh}$.

To conclude, modulator schemes that rely on the integration of efficient electro-optic materials with $\mathrm{SiPh}$ are gaining rapid technological maturity. They provide an additional route to fulfill the compelling needs of bandwidth, loss, and linearity at low-power consumption and cost for future high-speed optical links. It is important to note that the modulators based on the integration of efficient electro-optic materials with $\mathrm{SiPh}$ will not necessarily cannibalize or replace the plasma dispersion modulators. It is a way to overcome bandwidth and loss limits that stem from the physics of the plasma dispersion effect. However they will come at the cost of a more complex fabrication process. For this reason, the optimized solution will depend on the application.

\section{Acknowledgments}

The authors would like to thank Prof. Christian Koos, Prof. Marco Romagnoli, Prof. Delphine Marris-Morini, and Ashwyn Srinivasan for their valuable comments. This research is partially funded by the ERC starting grant ELECTRIC.

\section{References}

1. M. Romagnoli et al., "Graphene-based integrated photonics for next-generation datacom and telecom," Nat. Rev. Mater. 3(10), 392-414 (2018).

2. J. Witzens, "High-speed silicon photonics modulators," Proc. IEEE 106(12), 2158-2182 (2018).

3. B. Milivojevic et al., " $112 \mathrm{~Gb} / \mathrm{s}$ DP-QPSK transmission over 2427-km SSMF using small-size silicon photonic IQ modulator and low-power CMOS driver," in Opt. Fiber Commun. Conf. and Exposition and Natl. Fiber Opt. Eng. Conf., OTh1D.1 (2013).

4. S. S. Azadeh et al., "Low $V_{\pi}$ silicon photonics modulators with highly linear epitaxially grown phase shifters," Opt. Express 23(18), 23526-23550 (2015).

5. M. Webster et al., "Low-power MOS-capacitor based silicon photonic modulators and CMOS drivers," in Opt. Fiber Commun. Conf. and Exhib., W4H.3 (2015).

6. A. Narasimha et al., "A 40-Gb/s QSFP optoelectronic transceiver in a $0.13-\mu \mathrm{m}$ CMOS silicon-on-insulator technology," in Conf. Opt. Fiber Commun./Natl. Fiber Opt. Eng. Conf., OMK7 (2008).

7. Y. Sobu et al., "70 Gbaud operation of all-silicon Mach-Zehnder modulator based on forward-biased PIN diodes and passive equalizer," in 24th OptoElectron. and Commun. Conf. and Int. Conf. Photonics Switch. and Comput., MD2-2 (2019).

8. D. Patel et al., "High-speed compact silicon photonic Michelson interferometric modulator," Opt. Express 22(22), 26788-26802 (2014).

9. J. Sun et al., "A $128 \mathrm{~Gb} / \mathrm{s}$ PAM4 silicon microring modulator with integrated thermo-optic resonance tuning," J. Lightwave Technol. 37(1), 110-115 (2019).

10. K. Goi et al., "Silicon Mach-Zehnder modulator using low-loss phase shifter with bottom PN junction formed by restricted-depth doping," IEICE Electron. Express 10(17), 20130552 (2013).

11. X. Tu et al., "Silicon optical modulator with shield coplanar waveguide electrodes," Opt. Express 22(19), 23724-23731 (2014). 
12. J. Van Campenhout et al., "Low-voltage, low-loss, multi-Gb/s silicon micro-ring modulator based on a MOS capacitor," in Opt. Fiber Commun. Conf., OM2E.4 (2012).

13. X. Xiao et al., "High-speed, low-loss silicon Mach-Zehnder modulators with doping optimization," Opt. Express 21(4), 4116-4125 (2013).

14. M. Li et al., "Silicon intensity Mach-Zehnder modulator for single lane $100 \mathrm{~Gb} / \mathrm{s}$ applications," Photonics Res. 6(2), 109-116 (2018).

15. X. Tu et al., "50-Gb/s silicon optical modulator with travelingwave electrodes," Opt. Express 21(10), 12776-12782 (2013).

16. D. J. Thomson et al., "50-Gb/s silicon optical modulator," IEEE Photonics Technol. Lett. 24(4), 234-236 (2012).

17. D. Patel et al., "Design, analysis, and transmission system performance of a $41 \mathrm{GHz}$ silicon photonic modulator," Opt. Express 23(11), 14263-14287 (2015).

18. E. Timurdogan et al., "An ultralow power athermal silicon modulator," Nat. Commun. 5(1), 4008 (2014).

19. J. Ding et al., "Electro-optical response analysis of a $40 \mathrm{~Gb} / \mathrm{s}$ silicon Mach-Zehnder optical modulator," J. Lightwave Technol. 31(14), 2434-2440 (2013).

20. S. A. Srinivasan et al., "50 Gb/s C-band GeSi waveguide electro-absorption modulator," in Opt. Fiber Commun. Conf., Tu3D.7 (2016).

21. G. T. Reed et al., "Recent breakthroughs in carrier depletion based silicon optical modulators," Nanophotonics 3(4-5), 229245 (2014).

22. D. Petousi et al., "Monolithically integrated high-extinction-ratio MZM with a segmented driver in photonic BiCMOS," IEEE Photonics Technol. Lett. 28(24), 2866-2869 (2016).

23. A. Chen and E. E. Murphy, Broadband Optical Modulators: Science, Technology, and Applications, 1st ed., CRC Press, Boca Raton, Florida (2012).

24. S. K. Kim et al., "Theoretical and experimental study of $10 \mathrm{~Gb} / \mathrm{s}$ transmission performance using $1.55-\mu \mathrm{m} \mathrm{LiNbO}_{3}$-based transmitters with adjustable extinction ratio and chirp," J. Lightwave Technol. 17(8), 1320-1325 (1999).

25. N. Qi et al., "Co-design and demonstration of a $25-\mathrm{Gb} / \mathrm{s}$ siliconphotonic Mach-Zehnder modulator with a CMOS-based highswing driver," IEEE J. Sel. Top. Quantum Electron. 22(6), 131-140 (2016).

26. L. Chen, P. Dong, and Y. Chen, "Chirp and dispersion tolerance of a single-drive push-pull silicon modulator at $28 \mathrm{~Gb} / \mathrm{s}$," IEEE Photonics Technol. Lett. 24(11), 936-938 (2012).

27. M. Jacques et al., "Modulator material impact on chirp, DSP, and performance in coherent digital links: comparison of the lithium niobate, indium phosphide, and silicon platforms," Opt. Express 26(17), 22471-22490 (2018).

28. K. Goi et al., "20-Gbps BPSK silicon Mach-Zehnder modulator with excellent chirp-free performance," in 17th Opto-Electron. and Commun. Conf., pp. 238-239 (2012).

29. R. Li et al., "High-speed low-chirp PAM-4 transmission based on push-pull silicon photonic microring modulators," Opt. Express 25(12), 13222-13229 (2017).

30. K. Goi et al., "11-Gb/s 80-km transmission performance of zerochirp silicon Mach-Zehnder modulator," Opt. Express 20(26), B350-B356 (2012).

31. J. Liu et al., "Waveguide-integrated, ultralow-energy $\mathrm{GeSi}$ electro-absorption modulators," Nat. Photonics 2(7), 433-437 (2008).

32. D. Marris-Morini et al., "Low loss $40 \mathrm{Gbit} / \mathrm{s}$ silicon modulator based on interleaved junctions and fabricated on $300 \mathrm{~mm}$ SOI wafers," Opt. Express 21(19), 22471-22475 (2013).

33. J. C. Rosenberg et al., "A 25 Gbps silicon microring modulator based on an interleaved junction," Opt. Express 20(24), 2641126423 (2012).

34. D. A. B. Miller, "Device requirements for optical interconnects to silicon chips," Proc. IEEE 97(7), 1166-1185 (2009).
35. Q. Cheng et al., "Recent advances in optical technologies for data centers: a review," Optica 5(11), 1354-1370 (2018).

36. M. Asghari and A. V. Krishnamoorthy, "Energy-efficient communication," Nat. Photonics 5(5), 268-270 (2011).

37. F. Boeuf et al., "Benchmarking Si, SiGe, and III-V/Si hybrid SIS optical modulators for datacenter applications," J. Lightwave Technol. 35(18), 4047-4055 (2017).

38. N.-N. Feng et al., "30 GHz Ge electro-absorption modulator integrated with $3 \mu \mathrm{m}$ silicon-on-insulator waveguide," Opt. Express 19(8), 7062-7067 (2011).

39. A. Rahim et al., "Open-access silicon photonics: current status and emerging initiatives," Proc. IEEE 106(12), 2313-2330 (2018).

40. A. Meighan et al., "Design of $100 \mathrm{GHz}$-class Mach-Zehnder modulators in a generic indium phosphide platform," in IEEE Photonics Conf. (2020).

41. S. Lange et al., " $100 \mathrm{GBd}$ intensity modulation and direct detection with an InP-based monolithic DFB laser Mach-Zehnder modulator," J. Lightwave Technol. 36(1), 97-102 (2018).

42. M. Smit, K. Williams, and J. van der Tol, "Past, present, and future of InP-based photonic integration," APL Photonics 4(5), 050901 (2019).

43. K. Luke et al., "Wafer-scale low-loss lithium niobate photonic integrated circuits," Opt. Express 28(17), 24452-24458 (2020).

44. P. De Dobbelaere et al., "Advanced silicon photonics technology platform leveraging a semiconductor supply chain," in IEEE Int. Electron Dev. Meeting, pp. 34.1.1-34.1.4 (2017).

45. D. J. Shin et al., "Integration of silicon photonics into DRAM process," in Opt. Fiber Commun. Conf./Natl. Fiber Opt. Eng. Conf., OTu2C.4 (2013).

46. R. Meade et al., "TeraPHY: a high-density electronic-photonic Chiplet for optical I/O from a multi-chip module," in Opt. Fiber Commun. Conf., M4D.7 (2019).

47. F. Boeuf et al., "Recent progress in silicon photonics R\&D and manufacturing on $300 \mathrm{~mm}$ wafer platform," in Opt. Fiber Commun. Conf., W3A.1 (2015).

48. S. Fathololoumi et al., "1.6Tbps silicon photonics integrated circuit for co-packaged optical-IO switch applications," in Opt. Fiber Commun. Conf., T3H.1 (2020).

49. X. Chen et al., "The emergence of silicon photonics as a flexible technology platform," Proc. IEEE 106(12), 2101-2116 (2018).

50. C. Doerr et al., "Single-chip silicon photonics $100-\mathrm{Gb} / \mathrm{s}$ coherent transceiver," in Opt. Fiber Commun. Conf., Th5C.1 (2014).

51. J. C. Rosenberg et al., "Low-power 30 Gbps silicon microring modulator," in CLEO: Laser Appl. Photonic Appl., PDPB9 (2011).

52. C. Xiong et al., "Monolithic $56 \mathrm{~Gb} / \mathrm{s}$ silicon photonic pulseamplitude modulation transmitter," Optica 3(10), 1060-1065 (2016).

53. "Cisco annual internet report (2018-2023) white paper-Cisco," https://www.cisco.com/c/en/us/solutions/collateral/executiveperspectives/annual-internet-report/white-paper-c11-741490.html (accessed 16 July 2020).

54. P. J. Winzer, D. T. Neilson, and A. R. Chraplyvy, "Fiber-optic transmission and networking: the previous 20 and the next 20 years [Invited]," Opt. Express 26(18), 24190-24239 (2018).

55. P. Dong et al., " $50-\mathrm{Gb} / \mathrm{s}$ silicon quadrature phase-shift keying modulator," Opt. Express 20(19), 21181-21186 (2012).

56. P. Dong et al., "Experimental demonstration of microring quadrature phase-shift keying modulators," Opt. Lett. 37(7), 11781180 (2012).

57. "802.3bs-2017-IEEE Standard for Ethernet Amendment 10: media access control parameters, physical layers, and management parameters for $200 \mathrm{~Gb} / \mathrm{s}$ and $400 \mathrm{~Gb} / \mathrm{s}$ operation," https:// standards.ieee.org/standard/802_3bs-2017.html (accessed 16 July 2020).

58. X. Zhou, R. Urata, and H. Liu, "Beyond $1 \mathrm{~Tb} / \mathrm{s}$ intra-data center interconnect technology: IM-DD OR coherent?" J. Lightwave Technol. 38(2), 475-484 (2020). 
59. B. Jalali and S. Fathpour, "Silicon photonics," J. Lightwave Technol. 24(12), 4600-4615 (2006).

60. W. Bogaerts et al., "Nanophotonic waveguides in silicon-oninsulator fabricated with CMOS technology," J. Lightwave Technol. 23(1), 401-412 (2005).

61. R. Soref and B. Bennett, "Electrooptical effects in silicon," IEEE J. Quantum Electron. 23(1), 123-129 (1987).

62. Q. Xu et al., "Micrometre-scale silicon electro-optic modulator," Nature 435(7040), 325-327 (2005).

63. G. T. Reed and C. J. Png, "Silicon optical modulators," Mater. Today 8(1), 40-50 (2005).

64. C. K. Tang and G. T. Reed, "Highly efficient optical phase modulator in SOI waveguides," Electron. Lett. 31(6), 451-452 (1995).

65. T. Baba et al., "25-Gb/s broadband silicon modulator with $0.31-\mathrm{V} \cdot \mathrm{cm} V_{\pi} L$ based on forward-biased PIN diodes embedded with passive equalizer," Opt. Express 23(26), 32950-32960 (2015).

66. S. Tanaka et al., "Ultralow-power (1.59 mW/Gbps), 56-Gbps PAM4 operation of Si photonic transmitter integrating segmented PIN Mach-Zehnder modulator and 28-nm CMOS driver," J. Lightwave Technol. 36(5), 1275-1280 (2018).

67. S. Akiyama et al., "High-performance silicon modulator for integrated transceivers fabricated on 300-mm wafer," in Eur. Conf. Opt. Commun., P.2.8 (2014).

68. M. Webster et al., "An efficient MOS-capacitor based silicon modulator and CMOS drivers for optical transmitters," in 11th Int. Conf. Group IV Photonics, WB1 (2014).

69. J. Fujikata et al., "High-performance MOS-capacitor-type Si optical modulator and surface-illumination-type Ge photodetector for optical interconnection," Jpn. J. Appl. Phys. 55(4S), 04EC01 (2016).

70. M. Sodagar et al., "Compact, $15 \mathrm{~Gb} / \mathrm{s}$ electro-optic modulator through carrier accumulation in a hybrid $\mathrm{Si} / \mathrm{SiO}_{2} / \mathrm{Si}$ microdisk," Opt. Express 23(22), 28306-28315 (2015).

71. H. Yi et al., "Demonstration of low power penalty of silicon Mach-Zehnder modulator in long-haul transmission," Opt. Express 20(25), 27562-27568 (2012).

72. J.-B. You et al., " 12.5 Gbps optical modulation of silicon racetrack resonator based on carrier-depletion in asymmetric P-N diode," Opt. Express 16(22), 18340-18344 (2008).

73. D. J. Thomson et al., "High speed silicon optical modulator with self aligned fabrication process," Opt. Express 18(18), 1906419069 (2010).

74. D. J. Thomson et al., "High performance Mach-Zehnder-based silicon optical modulators," IEEE J. Sel. Top. Quantum Electron. 19(6), 85-94 (2013).

75. N.-N. Feng et al., "High speed carrier-depletion modulators with $1.4 \mathrm{~V}-\mathrm{cm} V_{\pi} L$ integrated on $0.25 \mu \mathrm{m}$ silicon-on-insulator waveguides," Opt. Express 18(8), 7994-7999 (2010).

76. P. Dong et al., "Low $\mathrm{V}_{\mathrm{pp}}$, ultralow-energy, compact, high-speed silicon electro-optic modulator," Opt. Express 17(25), 2248422490 (2009).

77. D. J. Thomson et al., "High contrast $40 \mathrm{Gbit} / \mathrm{s}$ optical modulation in silicon," Opt. Express 19(12), 11507-11516 (2011).

78. D. Marris-Morini et al., "Low loss and high speed silicon optical modulator based on a lateral carrier depletion structure," Opt. Express 16(1), 334-339 (2008).

79. M. Ziebell et al., " $40 \mathrm{Gbit} / \mathrm{s}$ low-loss silicon optical modulator based on a pipin diode," Opt. Express 20(10), 10591-10596 (2012).

80. H. Xu et al., "High speed silicon Mach-Zehnder modulator based on interleaved PN junctions," Opt. Express 20(14), 15093-15099 (2012).

81. H. Yu, W. Bogaerts, and A. De Keersgieter, "Optimization of ion implantation condition for depletion-type silicon optical modulators," IEEE J. Quantum Electron. 46(12), 1763-1768 (2010).
82. H. Xu et al., " 44 Gbit/s silicon Mach-Zehnder modulator based on interleaved PN junctions," in 9th Int. Conf. Group IV Photonics, pp. 201-203 (2012).

83. Z.-Y. Li et al., "Silicon waveguide modulator based on carrier depletion in periodically interleaved PN junctions," Opt. Express 17(18), 15947-15958 (2009).

84. M. Ziebell et al., "Ten Gbit/s ring resonator silicon modulator based on interdigitated PN junctions," Opt. Express 19(15), 14690-14695 (2011).

85. F. Y. Gardes et al., " $40 \mathrm{~Gb} / \mathrm{s}$ silicon photonics modulator for TE and TM polarisations," Opt. Express 19(12), 11804-11814 (2011).

86. X. Xiao et al., "44-Gb/s silicon microring modulators based on zigzag PN junctions," IEEE Photonics Technol. Lett. 24(19), 1712-1714 (2012).

87. X. Xiao et al., "High speed silicon photonic modulators," in Opt. Fiber Commun. Conf., Tu2H.1 (2017).

88. A. Irace, G. Breglio, and A. Cutolo, "All-silicon optoelectronic modulator with $1 \mathrm{GHz}$ switching capability," Electron. Lett. 39(2), 232-233 (2003).

89. R. Soref and J. Larenzo, "All-silicon active and passive guidedwave components for $\lambda=13$ and $1.6 \mu \mathrm{m}$," IEEE J. Quantum Electron. 22(6), 873-879 (1986)

90. A. Cutolo et al., "Silicon electro-optic modulator based on a three terminal device integrated in a low-loss single-mode SOI waveguide," J. Lightwave Technol. 15(3), 505-518 (1997).

91. S. Akiyama et al., "Compact PIN-diode-based silicon modulator using side-wall-grating waveguide," IEEE J. Sel. Top. Quantum Electron. 19(6), 74-84 (2013).

92. S. J. Spector et al., "High-speed silicon electro-optical modulator that can be operated in carrier depletion or carrier injection mode," in Conf. Lasers and Electro-Opt. and Conf. Quantum Electron. and Laser Sci., CFH4 (2008).

93. A. Sciuto et al., "Design, fabrication, and testing of an integrated Si-based light modulator," J. Lightwave Technol. 21(1), 228-235 (2003).

94. S. J. Spector et al., "Operation and optimization of silicon-diodebased optical modulators," IEEE J. Sel. Top. Quantum Electron. 16(1), 165-172 (2010).

95. J. C. Rosenberg et al., "Ultra-low-voltage micro-ring modulator integrated with a CMOS feed-forward equalization driver," in Opt. Fiber Commun. Conf./Natl. Fiber Opt. Eng. Conf., OWQ4 (2011).

96. C. E. Png et al., "Optical phase modulators for $\mathrm{MHz}$ and $\mathrm{GHz}$ modulation in silicon-on-insulator (SOI)," J. Lightwave Technol. 22(6), 1573-1582 (2004).

97. Q. Xu et al., "12.5 Gbit/s carrier-injection-based silicon microring silicon modulators," Opt. Express 15(2), 430-436 (2007).

98. A. Shakoor et al., "Compact 1D-silicon photonic crystal electrooptic modulator operating with ultra-low switching voltage and energy," Opt. Express 22(23), 28623-28634 (2014).

99. W. M. J. Green et al., "Ultra-compact, low RF power, $10 \mathrm{~Gb} / \mathrm{s}$ silicon Mach-Zehnder modulator," Opt. Express 15(25), 1710617113 (2007)

100. S. Meister et al., "High-speed Fabry-Pérot optical modulator in silicon with 3- $\mu$ m diode," J. Lightwave Technol. 33(4), 878-881 (2015).

101. L. Liao et al., "High speed silicon Mach-Zehnder modulator," Opt. Express 13(8), 3129-3135 (2005).

102. A. Liu et al., "A high-speed silicon optical modulator based on a metal-oxide-semiconductor capacitor," Nature 427(6975), 615618 (2004).

103. K. Debnath et al., "All-silicon carrier accumulation modulator based on a lateral metal-oxide-semiconductor capacitor," Photonics Res. 6(5), 373-379 (2018).

104. A. Abraham et al., "Evaluation of the performances of a silicon optical modulator based on a silicon-oxide-silicon capacitor," in 11th Int. Conf. Group IV Photonics, WB2 (2014). 
105. E. Li et al., "One-volt silicon photonic crystal nanocavity modulator with indium oxide gate," Opt. Lett. 43(18), 4429-4432 (2018).

106. L. Liao et al., " $40 \mathrm{Gbit} / \mathrm{s}$ silicon optical modulator for highspeed applications," Electron. Lett. 43(22), 1196-1197 (2007).

107. Y. Maegami et al., "High-efficiency strip-loaded waveguide based silicon Mach-Zehnder modulator with vertical p-n junction phase shifter," Opt. Express 25(25), 31407-31416 (2017).

108. A. Liu et al., "High-speed optical modulation based on carrier depletion in a silicon waveguide," Opt. Express 15(2), 660-668 (2007).

109. F. Y. Gardes et al., "A sub-micron depletion-type photonic modulator in silicon on insulator," Opt. Express 13(22), 8845-8854 (2005).

110. M. R. Watts et al., "Low-voltage, compact, depletion-mode, silicon Mach-Zehnder modulator," IEEE J. Sel. Top. Quantum Electron. 16(1), 159-164 (2010).

111. M. R. Watts et al., "Vertical junction silicon microdisk modulators and switches," Opt. Express 19(22), 21989-22003 (2011).

112. H. C. Nguyen et al., "Compact and fast photonic crystal silicon optical modulators," Opt. Express 20(20), 22465-22474 (2012).

113. Y. Terada et al., "Full C-band Si photonic crystal waveguide modulator," Opt. Lett. 42(24), 5110-5112 (2017).

114. Y. Hinakura, H. Arai, and T. Baba, "64 Gbps Si photonic crystal slow light modulator by electro-optic phase matching," Opt. Express 27(10), 14321-14327 (2019).

115. R. Dubé-Demers, S. LaRochelle, and W. Shi, "Ultrafast pulseamplitude modulation with a femtojoule silicon photonic modulator," Optica 3(6), 622-627 (2016).

116. P. Dong, L. Chen, and Y. K. Chen, "High-speed low-voltage single-drive push-pull silicon Mach-Zehnder modulators," Opt. Express 20(6), 6163-6169 (2012).

117. M. Streshinsky et al., "Low power $50 \mathrm{~Gb} / \mathrm{s}$ silicon traveling wave Mach-Zehnder modulator near $1300 \mathrm{~nm}$," Opt. Express 21(25), 30350-30357 (2013).

118. T. Baehr-Jones et al., "Ultralow drive voltage silicon travelingwave modulator," Opt. Express 20(11), 12014-12020 (2012).

119. R. Ding et al., "A compact low-power 320-Gb/s WDM transmitter based on silicon microrings," IEEE Photonics J. 6(3), 6600608 (2014).

120. X. Tu et al., "Fabrication of low loss and high speed silicon optical modulator using doping compensation method," Opt. Express 19(19), 18029-18035 (2011).

121. X. Li et al., "Highly efficient silicon Michelson interferometer modulators," IEEE Photonics Technol. Lett. 25(5), 407-409 (2013).

122. K. Li et al., "Electronic-photonic convergence for silicon photonics transmitters beyond 100 Gbps on-off keying," Optica 7(11), 1514-1516 (2020).

123. H. Zhang et al., "800 Gbit/s transmission over $1 \mathrm{~km}$ single-mode fiber using a four-channel silicon photonic transmitter," Photonics Res. 8(11), 1776-1782 (2020).

124. D. Pérez-Galacho et al., "QPSK modulation in the O-band using a single dual-drive Mach-Zehnder silicon modulator," J. Lightwave Technol. 36(18), 3935-3940 (2018).

125. F. Fresi et al., "Silicon photonics integrated 16-QAM modulator exploiting only binary driving electronics," in Opt. Fiber Commun. Conf., Th3J.6 (2016).

126. S. Zhalehpour et al., "All-silicon IQ modulator for $100 \mathrm{GBaud}$ 32QAM transmissions," in Opt. Fiber Commun. Conf. Postdeadline Papers, Th4A.5 (2019).

127. L. Deniel et al., "DAC-less PAM-4 generation in the O-band using a silicon Mach-Zehnder modulator," Opt. Express 27(7), 9740-9748 (2019).

128. Y. Kim et al., "Strain-induced enhancement of plasma dispersion effect and free-carrier absorption in SiGe optical modulators," Sci. Rep. 4(1), 4683 (2014).
129. C. G. Bottenfield, V. A. Thomas, and S. E. Ralph, "Silicon photonic modulator linearity and optimization for microwave photonic links," IEEE J. Sel. Top. Quantum Electron. 25(5), 3400110 (2019).

130. L. Chrostowski et al., "Impact of fabrication non-uniformity on chip-scale silicon photonic integrated circuits," in Opt. Fiber Commun. Conf., Th2A.37 (2014).

131. W. Bogaerts et al., "Silicon microring resonators," Laser Photonics Rev. 6(1), 47-73 (2012).

132. B. Guha, B. B. C. Kyotoku, and M. Lipson, "CMOS-compatible athermal silicon microring resonators," Opt. Express 18(4), 3487-3493 (2010).

133. D. Feng et al., "High-speed GeSi electroabsorption modulator on the SOI waveguide platform," IEEE J. Sel. Top. Quantum Electron. 19(6), 64-73 (2013).

134. S. Gupta et al., "50-GHz Ge waveguide electro-absorption modulator integrated in a $220 \mathrm{~nm}$ SOI photonics platform," in Opt. Fiber Commun. Conf., Tu2A.4 (2015).

135. D. Feng et al., "High speed GeSi electro-absorption modulator at $1550 \mathrm{~nm}$ wavelength on SOI waveguide," Opt. Express 20(20), 22224-22232 (2012).

136. S. A. Srinivasan et al., " $56 \mathrm{~Gb} / \mathrm{s}$ Germanium waveguide electroabsorption modulator," J. Lightwave Technol. 34(2), 419-424 (2016).

137. J. Verbist et al., " $100 \mathrm{~Gb} / \mathrm{s}$ DAC-less and DSP-free transmitters using GeSi EAMs for short-reach optical interconnects," in Opt. Fiber Commun. Conf., W4D.4 (2018).

138. A. E.-J. Lim et al., "Novel evanescent-coupled germanium electro-absorption modulator featuring monolithic integration with germanium $\mathrm{p}-\mathrm{i}-\mathrm{n}$ photodetector," Opt. Express 19(6), 5040-5046 (2011)

139. L. Mastronardi et al., "High-speed $\mathrm{Si} / \mathrm{GeSi}$ hetero-structure electro absorption modulator," Opt. Express 26(6), 6663-6673 (2018).

140. Y. Tang, J. D. Peters, and J. E. Bowers, "Over $67 \mathrm{GHz}$ bandwidth hybrid silicon electroabsorption modulator with asymmetric segmented electrode for $1.3 \mu \mathrm{m}$ transmission," Opt. Express 20(10), 11529-11535 (2012).

141. P. Chaisakul et al., "Integrated germanium optical interconnects on silicon substrates," Nat. Photonics 8(6), 482-488 (2014).

142. P. Chaisakul et al., "Recent progress in GeSi electro-absorption modulators," J. Lightwave Technol. 15(1), 014601 (2014).

143. J. Frigerio et al., "Giant electro-optic effect in Ge/SiGe coupled quantum wells," Sci. Rep. 5(1), 15398 (2015).

144. Y.-H. Kuo et al., "Strong quantum-confined Stark effect in germanium quantum-well structures on silicon," Nature 437(7063), 1334-1336 (2005).

145. D. A. B. Miller et al., "Band-edge electroabsorption in quantum well structures: the quantum-confined Stark effect," Phys. Rev. Lett. 53(22), 2173-2176 (1984).

146. D. A. B. Miller, "Attojoule optoelectronics for low-energy information processing and communications," J. Lightwave Technol. 35(3), 346-396 (2017).

147. S. Ren et al., "Ge/SiGe quantum well waveguide modulator monolithically integrated with SOI waveguides," IEEE Photonics Technol. Lett. 24(6), 461-463 (2012).

148. P. Chaisakul et al., " $23 \mathrm{GHz} \mathrm{Ge} / \mathrm{SiGe}$ multiple quantum well electro-absorption modulator," Opt. Express 20(3), 3219-3224 (2012).

149. R. M. Audet et al., "Surface-normal Ge/SiGe asymmetric FabryPerot optical modulators fabricated on silicon substrates," J. Lightwave Technol. 31(24), 3995-4003 (2013).

150. E. H. Edwards et al., "Ge/SiGe asymmetric Fabry-Perot quantum well electroabsorption modulators," Opt. Express 20(28), 29164-29173 (2012).

151. P. Chaisakul et al., "Recent progress on $\mathrm{Ge} / \mathrm{SiGe}$ quantum well optical modulators, detectors, and emitters for optical interconnects," Photonics 6(1), 24 (2019). 
152. S. A. Srinivasan et al., "High absorption contrast quantum confined Stark effect in ultra-thin $\mathrm{Ge} / \mathrm{SiGe}$ quantum well stacks grown on Si," IEEE J. Quantum Electron. 56(1), 5200207 (2020).

153. M. Liu et al., "A graphene-based broadband optical modulator," Nature 474(7349), 64-67 (2011).

154. M. A. Giambra et al., "High-speed double layer graphene electroabsorption modulator on SOI waveguide," Opt. Express 27(15), 20145-20155 (2019).

155. M. Mohsin et al., "Graphene based low insertion loss electroabsorption modulator on SOI waveguide," Opt. Express 22(12), 15292-15297 (2014)

156. M. Liu, X. Yin, and X. Zhang, "Double-layer graphene optical modulator," Nano Lett. 12(3), 1482-1485 (2012).

157. Y. Hu et al., "Broadband $10 \mathrm{~Gb} / \mathrm{s}$ operation of graphene electroabsorption modulator on silicon," Laser Photonics Rev. 10(2), 307-316 (2016).

158. C. T. Phare et al., "Graphene electro-optic modulator with 30 GHz bandwidth," Nat. Photonics 9(8), 511-514 (2015).

159. L. A. Shiramin et al., "High extinction ratio hybrid graphenesilicon photonic crystal switch," IEEE Photonics Technol. Lett. 30(2), 157-160 (2018).

160. V. Sorianello et al., "Chirp management in silicon-graphene electro absorption modulators," Opt. Express 25(16), 1937119381 (2017).

161. J.-H. Han et al., "Efficient low-loss InGaAsP/Si hybrid MOS optical modulator," Nat. Photonics 11(8), 486-490 (2017).

162. J. Witzens, "Modulators make efficiency leap," Nat. Photonics 11(8), 459-462 (2017).

163. T. Hiraki et al., "Heterogeneously integrated III-V/Si MOS capacitor Mach-Zehnder modulator," Nat. Photonics 11(8), 482-485 (2017).

164. Q. $\mathrm{Li}$ et al., "Si racetrack modulator with III-V/Si hybrid MOS optical phase shifter," in 45th Eur. Conf. Opt. Commun. (2019).

165. Q. Li et al., "Efficient optical modulator by reverse-biased III-V/ Si hybrid MOS capacitor based on FK effect and carrier depletion," in Opt. Fiber Commun. Conf., M4A.2 (2019).

166. T. Komljenovic et al., "Photonic integrated circuits using heterogeneous integration on silicon," Proc. IEEE 106(12), 2246-2257 (2018).

167. L. Cao et al., "Hybrid amorphous silicon (a-Si:H)- $\mathrm{LiNbO}_{3}$ electro-optic modulator," Opt. Commun. 330, 40-44 (2014).

168. A. Rao et al., "Heterogeneous microring and Mach-Zehnder modulators based on lithium niobate and chalcogenide glasses on silicon," Opt. Express 23(17), 22746-22752 (2015).

169. L. Chen et al., "Hybrid silicon and lithium niobate electro-optical ring modulator," Optica 1(2), 112-118 (2014).

170. S. Jin et al., " $\mathrm{LiNbO}_{3}$ thin-film modulators using silicon nitride surface ridge waveguides," IEEE Photonics Technol. Lett. 28(7), 736-739 (2016).

171. A. Rao et al., "High-performance and linear thin-film lithium niobate Mach-Zehnder modulators on silicon up to $50 \mathrm{GHz}$," Opt. Lett. 41(24), 5700-5703 (2016).

172. N. Boynton et al., "A heterogeneously integrated silicon photonic/lithium niobate travelling wave electro-optic modulator," Opt. Express 28(2), 1868-1884 (2020).

173. P. O. Weigel et al., "Bonded thin film lithium niobate modulator on a silicon photonics platform exceeding $100 \mathrm{GHz} 3-\mathrm{dB}$ electrical modulation bandwidth," Opt. Express 26(18), 2372823739 (2018).

174. M. He et al., "High-performance hybrid silicon and lithium niobate Mach-Zehnder modulators for 100 Gbit s$^{-1}$ and beyond," Nat. Photonics 13(5), 359-364 (2019).

175. M. Li and H. X. Tang, "Strong Pockels materials," Nat. Mater. 18(1), 9-11 (2019)

176. S. Abel et al., "A hybrid barium titanate-silicon photonics platform for ultraefficient electro-optic tuning," J. Lightwave Technol. 34(8), 1688-1693 (2016).
177. C. Xiong et al., "Active silicon integrated nanophotonics: ferroelectric $\mathrm{BaTiO}_{3}$ devices," Nano Lett. 14(3), 1419-1425 (2014).

178. F. Eltes et al., "Low-loss $\mathrm{BaTiO}_{3}-\mathrm{Si}$ waveguides for nonlinear integrated photonics," ACS Photonics 3(9), 1698-1703 (2016).

179. S. Abel et al., "Large Pockels effect in micro- and nanostructured barium titanate integrated on silicon," Nat. Mater. 18(1), 42-47 (2019).

180. A. Messner et al., "Integrated ferroelectric $\mathrm{BaTiO}_{3} / \mathrm{Si}$ plasmonic modulator for $100 \mathrm{Gbit} / \mathrm{s}$ and beyond," in Opt. Fiber Commun. Conf., M2I.6 (2018).

181. F. Eltes et al., "A novel 25 Gbps electro-optic Pockels modulator integrated on an advanced Si photonic platform," in IEEE Int. Electron Dev. Meeting, pp. 24.5.1-24.5.4 (2017).

182. F. Eltes et al., "A $\mathrm{BaTiO}_{3}$-based electro-optic Pockels modulator monolithically integrated on an advanced silicon photonics platform," J. Lightwave Technol. 37(5), 1456-1462 (2019).

183. K. Alexander et al., "Broadband electro-optic modulation using low-loss PZT-on-silicon nitride integrated waveguides," in Conf. Lasers and Electro-Opt., JTh5C.7 (2017).

184. K. Alexander et al., "Nanophotonic Pockels modulators on a silicon nitride platform," Nat Commun 9(1), 3444 (2018).

185. K. Alexander, "Integrated silicon nitride photonics with highly nonlinear thin films and 2D materials: properties and devices," $\mathrm{PhD}$ thesis, Ghent University (2018).

186. C. Koos et al., "All-optical high-speed signal processing with silicon-organic hybrid slot waveguides," Nat. Photonics 3(4), 216-219 (2009).

187. D. Korn et al., "Lasing in silicon-organic hybrid waveguides," Nat. Commun. 7(1), 10864 (2016).

188. C. Koos et al., "Silicon-organic hybrid (SOH) and plasmonicorganic hybrid (POH) integration," J. Lightwave Technol. 34(2), 256-268 (2016).

189. R. Palmer et al., "High-speed, low drive-voltage siliconorganic hybrid modulator based on a binary-chromophore electro-optic material," J. Lightwave Technol. 32(16), 27262734 (2014).

190. C. Kieninger et al., "Ultra-high electro-optic activity demonstrated in a silicon-organic hybrid modulator," Optica 5(6), 739-748 (2018).

191. L. Alloatti et al., "100 GHz silicon-organic hybrid modulator," Light Sci. Appl. 3(5), e173 (2014).

192. S. Koeber et al., "Femtojoule electro-optic modulation using a silicon-organic hybrid device," Light Sci. Appl. 4(2), e255 (2015).

193. H. Zwickel et al., "Silicon-organic hybrid (SOH) modulators for intensity-modulation/direct-detection links with line rates of up to $120 \mathrm{Gbit} / \mathrm{s}$," Opt. Express 25(20), 23784-23800 (2017).

194. C. Kieninger et al., "Silicon-organic hybrid (SOH) MachZehnder modulators for 100 GBd PAM4 signaling with sub1 dB phase-shifter loss," Opt. Express 28(17), 24693-24707 (2020).

195. C. Kieninger et al., "Demonstration of long-term thermally stable silicon-organic hybrid modulators at $85^{\circ} \mathrm{C}$," Opt. Express $\mathbf{2 6}(21)$, 27955-27964 (2018).

196. S. Wolf et al., "Silicon-organic hybrid (SOH) Mach-Zehnder modulators for $100 \mathrm{Gbit} / \mathrm{s}$ on-off keying," Sci. Rep. 8(1), 2598 (2018).

197. T. Baehr-Jones et al., "Optical modulation and detection in slotted Silicon waveguides," Opt. Express 13(14), 5216-5226 (2005).

198. H. Figi et al., "Electro-optic modulation in horizontally slotted silicon/organic crystal hybrid devices," J. Opt. Soc. Am. B 28(9), 2291-2300 (2011).

199. D. Korn et al., "Electro-optic organic crystal silicon high-speed modulator," IEEE Photonics J. 6(2), 2700109 (2014).

200. S. Ummethala et al., "Capacitively coupled silicon-organic hybrid modulator for 200 Gbit/s PAM-4 signaling," in Conf. Lasers and Electro-Opt., JTh5B.2 (2019). 
201. V. Sorianello et al., "Graphene-silicon phase modulators with gigahertz bandwidth," Nat. Photonics 12(1), 40-44 (2018).

202. M. Midrio et al., "Graphene-based optical phase modulation of waveguide transverse electric modes," Photonics Res. 2(3), A34A40 (2014).

203. H. Dalir et al., "Athermal broadband graphene optical modulator with 35 GHz speed," ACS Photonics 3(9), 1564-1568 (2016).

204. I. Datta et al., "Low-loss composite photonic platform based on 2D semiconductor monolayers," Nat. Photonics 14(4), 256-262 (2020).

205. S. Ye et al., "High-speed optical phase modulator based on graphene-silicon waveguide," IEEE J. Sel. Top. Quantum Electron. 23(1), 76-80 (2017).

206. C. Xu et al., "Characteristics of electro-refractive modulating based on graphene-oxide-silicon waveguide," Opt. Express 20(20), 22398-22405 (2012).

207. M. Mohsin et al., "Experimental verification of electro-refractive phase modulation in graphene," Sci. Rep. 5(1), 10967 (2015).

208. L. Yang et al., "Low-chirp high-extinction-ratio modulator based on graphene-silicon waveguide," Opt. Lett. 38(14), 2512-2515 (2013).

209. J. Leuthold et al., "High-speed, low-power optical modulators in silicon," in 15th Int. Conf. Transp. Opt. Networks, We.D2.1 (2013).

210. A. Moscoso-Mártir et al., "Co-integration of a temperature tolerant low impedance resonantly enhanced silicon photonics modulator," in IEEE 14th Int. Conf. Group IV Photonics, pp. 101-102 (2017).

211. X. Li et al., "Single-drive high-speed lumped depletion-type modulators toward $10 \mathrm{fJ} /$ bit energy consumption," Photonics Res. 5(2), 134-142 (2017).

212. A. Giuglea et al., "Comparison of segmented and traveling-wave electro-optical transmitters based on silicon photonics MachZehnder modulators," in Photonics Switching and Comput. (2018).

213. S. Lin et al., "Electronic-photonic co-optimization of high-speed silicon photonic transmitters," J. Lightwave Technol. 35(21), 4766-4780 (2017).

214. D. J. Thomson et al., "Optical detection and modulation at $2 \mu \mathrm{m}-2.5 \mu \mathrm{m}$ in silicon," Opt. Express 22(9), 10825-10830 (2014).

215. M. Montesinos-Ballester et al., "Optical modulation in Ge-rich $\mathrm{SiGe}$ waveguides in the mid-infrared wavelength range up to $11 \mu \mathrm{m}, "$ Commun. Mater. 1(1), 6 (2020).

216. J. P. Lorenzo and R. A. Soref, " $1.3 \mu \mathrm{m}$ electro-optic silicon switch," Appl. Phys. Lett. 51(1), 6-8 (1987).

217. B. Chmielak et al., "Pockels effect based fully integrated, strained silicon electro-optic modulator," Opt. Express 19(18), 17212-17219 (2011).

218. R. S. Jacobsen et al., "Strained silicon as a new electro-optic material," Nature 441(7090), 199-202 (2006).

219. P. Damas et al., "Wavelength dependence of Pockels effect in strained silicon waveguides," Opt. Express 22(18), 22095-22100 (2014).

220. Y. Shiraki et al., Silicon-Germanium (SiGe) Nanostructures, Y. Shiraki and N. Usami, Eds., Woodhead Publishing Series in Electronic and Optical Materials, Woodhead Publishing (2011).

221. L. Vivien and L. E. Pavesi, Handbook of Silicon Photonics, 1st ed., CRC Press (2013).

222. M. Zeiler et al., "Radiation damage in silicon photonic MachZehnder modulators and photodiodes," IEEE Trans. Nucl. Sci. 64(11), 2794-2801 (2017).

223. "Photonic integration and photonics-electronics convergence on silicon platform," https://www.frontiersin.org/research-topics/2972/ photonic-integration-and-photonics-electronics-convergence-onsilicon-platform (accessed 1 February 2021).

224. U. Chakraborty et al., "Cryogenic operation of silicon photonic modulators based on the DC Kerr effect," Optica 7(10), 13851390 (2020).
225. M. Gehl et al., "Operation of high-speed silicon photonic microdisk modulators at cryogenic temperatures," Optica 4(3), 374382 (2017).

226. S. M. Jackson et al., "A novel optical phase modulator design suitable for phased arrays," J. Lightwave Technol. 16(11), 2016-2019 (1998).

227. J. Shin et al., "Epitaxial growth technology for optical interconnect based on bulk-Si platform," in 10th Int. Conf. Group IV Photonics (2013).

228. O. Marshall et al., "Heterogeneous integration on silicon photonics," Proc. IEEE 106(12), 2258-2269 (2018).

229. S. Kodama, T. Yoshimatsu, and H. Ito, "500 Gbit/s optical gate monolithically integrating photodiode and electroabsorption modulator," Electron. Lett. 40(9), 555-556 (2004).

230. S. A. Srinivasan, "Advanced Germanium devices for optical interconnects," PhD Thesis, Ghent University (2017).

231. A. Melikyan et al., "Differential drive I/Q modulator based on silicon photonic electro-absorption modulators," J. Lightwave Technol. 38(11), 2872-2876 (2020).

232. Y. Tong et al., "Integrated germanium-on-silicon Franz-Keldysh vector modulator used with a Kramers-Kronig receiver," Opt. Lett. 43(18), 4333-4336 (2018).

233. J. Verbist et al., "Real-time and DSP-free 128 Gb/s PAM-4 link using a binary driven silicon photonic transmitter," J. Lightwave Technol. 37(2), 274-280 (2019).

234. K. M. Rabe et al., Modern Physics of Ferroelectrics: Essential Background, pp. 1-30, Springer (2007).

235. M. Veithen, X. Gonze, and P. Ghosez, "Nonlinear optical susceptibilities, Raman efficiencies, and electro-optic tensors from firstprinciples density functional perturbation theory," Phys. Rev. B 71(12), 125107 (2005).

236. H. Buhay et al., "Pulsed laser deposition and ferroelectric characterization of bismuth titanate films," Appl. Phys. Lett. 58(14), 1470-1472 (1991).

237. D. J. R. Appleby et al., "Ferroelectric properties in thin film barium titanate grown using pulsed laser deposition," J. Appl. Phys. 116(12), 124105 (2014).

238. T. Kobayashi et al., "Effect of multi-coating process on the orientation and microstructure of lead zirconate titanate (PZT) thin films derived by chemical solution deposition," Thin Solid Films 489(1), 74-78 (2005).

239. Z. Bi, Z. Zhang, and P. Fan, "Characterization of PZT ferroelectric thin films by RF-magnetron sputtering," J. Phys. Conf. Ser. 61, 120-124 (2007).

240. T. Li et al., "Metalorganic chemical vapor deposition of ferroelectric $\mathrm{SrBi}_{2} \mathrm{Ta}_{2} \mathrm{O}_{9}$ thin films," Appl. Phys. Lett. 68(5), 616-618 (1996).

241. F. Zhang et al., "Atomic layer deposition of $\mathrm{Pb}(\mathrm{Zr}, \mathrm{Ti}) \mathrm{O}_{\mathrm{x}}$ on $4 \mathrm{H}-\mathrm{SiC}$ for metal-ferroelectric-insulator-semiconductor diodes," J. Appl. Phys. 109(12), 124109 (2011).

242. E. L. Wooten et al., "A review of lithium niobate modulators for fiber-optic communications systems," IEEE J. Sel. Top. Quantum Electron. 6(1), 69-82 (2000).

243. M. Zhang et al., "Ultra-high bandwidth integrated lithium niobate modulators with record-low $V_{\pi}$," in Opt. Fiber Commun. Conf., Th4A.5 (2018).

244. K. Noguchi, O. Mitomi, and H. Miyazawa, "Millimeter-wave Ti: $\mathrm{LiNbO}_{3}$ optical modulators," J. Lightwave Technol. 16(4), 615-619 (1998).

245. G. Poberaj et al., "Lithium niobate on insulator (LNOI) for micro-photonic devices," Laser Photonics Rev. 6(4), 488-503 (2012).

246. A. J. Mercante et al., "Thin film lithium niobate electro-optic modulator with terahertz operating bandwidth," Opt. Express 26(11), 14810-14816 (2018).

247. C. Wang et al., "Integrated lithium niobate electro-optic modulators operating at CMOS-compatible voltages," Nature 562(7725), 101-104 (2018). 
248. H. Takagi and R. Maeda, "Room temperature bonding of silicon and lithium niobate," Appl. Phys. Lett. 89(3), 031914 (2006).

249. P. Rabiei et al., "Heterogeneous lithium niobate photonics on silicon substrates," Opt. Express 21(21), 25573-25581 (2013).

250. C. Wang et al., "Nanophotonic lithium niobate electro-optic modulators," Opt. Express 26(2), 1547-1555 (2018).

251. P. Bernasconi, M. Zgonik, and P. Günter, "Temperature dependence and dispersion of electro-optic and elasto-optic effect in perovskite crystals," J. Appl. Phys. 78(4), 2651-2658 (1995).

252. R. A. McKee et al., "Molecular beam epitaxy growth of epitaxial barium silicide, barium oxide, and barium titanate on silicon," Appl. Phys. Lett. 59(7), 782-784 (1991).

253. A. Petraru et al., "Ferroelectric $\mathrm{BaTiO}_{3}$ thin-film optical waveguide modulators," Appl. Phys. Lett. 81(8), 1375-1377 (2002).

254. A. A. Demkov and A. B. Posadas, Integration of Functional Oxides with Semiconductors, Springer, New York (2014).

255. M.-H. Hsu, "Monolithic integration of barium titanate on silicon for high-speed and power-efficient optical modulator applications," PhD Thesis, Ghent University (2016).

256. J. P. George et al., "Lanthanide-assisted deposition of strongly electro-optic PZT thin films on silicon: toward integrated active nanophotonic devices," ACS Appl. Mater. Interfaces 7(24), 13350-13359 (2015).

257. J. P. George, "Integration of ferroelectric thin films on silicon for electro-optic devices," PhD Thesis, Ghent University (2016).

258. W. Heni et al., "Silicon-organic and plasmonic-organic hybrid photonics," ACS Photonics 4(7), 1576-1590 (2017).

259. H. Zwickel et al., "Verified equivalent-circuit model for slotwaveguide modulators," Opt. Express 28(9), 12951-12976 (2020).

260. S. Wolf et al., "DAC-less amplifier-less generation and transmission of QAM signals using sub-volt silicon-organic hybrid modulators," J. Lightwave Technol. 33(7), 1425-1432 (2015).

261. S. Wolf et al., "Coherent modulation up to $100 \mathrm{GBd}$ 16QAM using silicon-organic hybrid (SOH) devices," Opt. Express 26(1), 220-232 (2018).

262. G.-W. Lu et al., "High-temperature-resistant silicon-polymer hybrid modulator operating at up to $200 \mathrm{Gbit} \mathrm{s}^{-1}$ for energyefficient datacentres and harsh-environment applications," Nat. Commun. 11(1), 4224 (2020).

263. M. Jazbinsek et al., "Organic electro-optic single crystalline films for integrated optics," Proc. SPIE 7774, $77740 \mathrm{Q}$ (2010).

264. A. Melikyan et al., "High-speed plasmonic phase modulators," Nat. Photonics 8(3), 229-233 (2014).

265. C. Haffner et al., "Plasmonic organic hybrid modulators-scaling highest speed photonics to the microscale," Proc. IEEE 104(12), 2362-2379 (2016).

266. C. Hoessbacher et al., "Plasmonic modulator with $170 \mathrm{GHz}$ bandwidth demonstrated at $100 \mathrm{GBd}$ NRZ," Opt. Express 25(3), 1762-1768 (2017).

267. C. Uhl, H. Hettrich, and M. Möller, “A $100 \mathrm{Gbit} / \mathrm{s} 2 \mathrm{~V}_{\mathrm{pp}}$ power multiplexer in SiGe BiCMOS technology for directly driving a monolithically integrated plasmonic MZM in a silicon photonics transmitter," in IEEE Bipolar/BiCMOS Circuits and Technol. Meeting, pp. 106-109 (2017).

268. W. Heni et al., "High speed plasmonic modulator array enabling dense optical interconnect solutions," Opt. Express 23(23), 29746-29757 (2015).

269. A. Melikyan et al., "Plasmonic-organic hybrid (POH) modulators for OOK and BPSK signaling at $40 \mathrm{Gbit} / \mathrm{s}$," Opt. Express 23(8), 9938-9946 (2015).

270. S. Ummethala et al., "THz-to-optical conversion in wireless communications using an ultra-broadband plasmonic modulator," Nat. Photonics 13(8), 519-524 (2019).

271. G. Duan et al., "Hybrid III-V on silicon lasers for photonic integrated circuits on silicon," IEEE J. Sel. Top. Quantum Electron. 20(4), 158-170 (2014).
272. D. Liang and J. E. Bowers, "Recent progress in lasers on silicon," Nat. Photonics 4(8), 511-517 (2010).

273. T. Shimizu et al., "High density hybrid integrated light source with a laser diode array on a silicon optical waveguide platform for inter-chip optical interconnection," in 8th IEEE Int. Conf. Group IV Photonics, pp. 181-183 (2011).

274. G. Roelkens et al., "III-V/Si PICs based on micro-transfer-printing," in Opt. Fiber Commun. Conf., W4E.6 (2019).

275. A. J. Zilkie et al., "Multi-micron silicon photonics platform for highly manufacturable and versatile photonic integrated circuits," IEEE J. Sel. Top. Quantum Electron. 25(5), 8200713 (2019).

276. B. Szelag et al., "Hybrid III-V/silicon technology for laser integration on a 200-mm fully CMOS-compatible silicon photonics platform," IEEE J. Sel. Top. Quantum Electron. 25(5), 8201210 (2019).

277. A. Alduino, "Demonstration of a high speed 4-channel integrated silicon photonics WDM link with hybrid silicon lasers," in IEEE Hot Chips 22 Symp., pp. 1-29 (2010).

278. S. Chen et al., "Electrically pumped continuous-wave III-V quantum dot lasers on silicon," Nat. Photonics 10(5), 307-311 (2016).

279. Z. Wang et al., "Room-temperature InP distributed feedback laser array directly grown on silicon," Nat. Photonics 9(12), 837-842 (2015).

280. Q. Li et al., "Optical phase modulators based on reverse-biased III-V/Si hybrid metal-oxide-semiconductor capacitors," IEEE Photonics Technol. Lett. 32(6), 345-348 (2020).

281. S. Ohno et al., "Taper-less III-V/Si hybrid MOS optical phase shifter using ultrathin InP membrane," in Opt. Fiber Commun. Conf., M2B.6 (2020).

282. Y. Tang et al., " $50 \mathrm{~Gb} / \mathrm{s}$ hybrid silicon traveling-wave electroabsorption modulator," Opt. Express 19(7), 5811-5816 (2011).

283. C. Mattevi, H. Kim, and M. Chhowalla, "A review of chemical vapour deposition of graphene on copper," J. Mater. Chem. 21(10), 3324-3334 (2011).

284. Y. S. Kim et al., "Direct growth of patterned graphene on $\mathrm{SiO}_{2}$ substrates without the use of catalysts or lithography," Nanoscale 6(17), 10100-10105 (2014).

285. X. Li et al., "Large-area synthesis of high-quality and uniform graphene films on copper foils," Science 324(5932), 1312-1314 (2009).

286. R. R. Nair et al., "Fine structure constant defines visual transparency of graphene," Science 320(5881), 1308 (2008).

287. K. I. Bolotin et al., "Ultrahigh electron mobility in suspended graphene," Solid State Commun. 146(9-10), 351-355 (2008).

288. A. S. Mayorov et al., "Micrometer-scale ballistic transport in encapsulated graphene at room temperature," Nano Lett. 11(6), 2396-2399 (2011).

289. V. Sorianello, M. Midrio, and M. Romagnoli, "Design optimization of single and double layer graphene phase modulators in SOI," Opt. Express 23(5), 6478-6490 (2015).

290. F. Wang et al., "Gate-variable optical transitions in graphene," Science 320(5873), 206-209 (2008).

291. L. A. Falkovsky, "Optical properties of graphene," J. Phys. Conf. Ser. 129, 012004 (2008).

Abdul Rahim is currently working with Professor Roel Baets at the Photonics Research Group of Ghent University. He is the coordinator of ePIXfab, the European Silicon Photonics Alliance. From 2009 to 2014, he worked in the research group of Professor Klaus Petermann and was awarded a doctorate degree by Technische Universitaet Berlin for his work on investigating innovative silicon photonic integrated filters for optical communication systems. Prior to his doctoral work, he worked in various capacities at the $R \& D$ departments of numerous public and private sector organizations.

Artur Hermans received his BSc degree in engineering (electronics and information technology) from Vrije Universiteit Brussel (VUB) in 2012, and the European MSc degree in photonics from VUB, Ghent 
University (UGent), and the University of St. Andrews in 2014. In 2019, he was awarded a PhD by Ghent University for his work on second-order nonlinear optical materials for silicon nitride photonic integrated circuits. Currently, he is working as a postdoctoral researcher in the Photonics Research Group at Ghent University-IMEC.

Benjamin Wohlfeil is currently a manager in the Advanced Technology Department at ADVA Optical Networking in Berlin and is involved in the photonic integration activities of ADVA. He received his Dipl.-Ing. degree in computer science from Technische Universität Berlin in 2008 and the Dr.-Ing. degree in electrical engineering also from Technische Universität Berlin in 2015. From 2014 to 2015, he worked as a postdoc in the Computational Nano-Optics Group at the Konrad-Zuse-Center for applied mathematics. He has authored more than a dozen papers and is inventor of several patents. $\mathrm{He}$ is an active contributor to OIF and COBO standardization groups regarding coherent optical transceivers and is principal investigator in several national and European research projects.

Despoina Petousi received her diploma in electrical and computer engineering from Aristotle University of Thessaloniki in 2009 where she received her master's degree in nanosciences and nanotechnologies in 2011. From 2011 until 2019 she was a researcher in the silicon photonics group of IHP, Leibniz Institut fuer Innovative Mikroelektronik, Frankfurt (Oder). In 2017, she received her PhD from Technische Universitä Berlin under the supervision of Professor Klaus Petermann. Her $\mathrm{PhD}$ thesis was focused on the development of integrated silicon photonic modulators with application in future coherent systems for optical communications. Currently, she is a research and development engineer in ADVA mainly focused on the field of photonic integration and next generation transceivers for optical interconnects.

Bart Kuyken received his BSc degree in physics and his BSc and MSc degrees in electrical engineering from Ghent University in 2006 and 2008, respectively, and his additional MSc degree from Stanford University in 2009. After obtaining his PhD from Ghent University in 2013, he focused his postdoctoral work on integrated nonlinear circuits and comb generation and was a visiting worker at the Max Planck Institute for Quantum Optics in 2013 and 2014. Currently, he is an associate professor at Ghent University, where he is looking at electrically pumped comb sources on chip.

Dries Van Thourhout received his MSc degree in physical engineering and his PhD from Ghent University, Ghent, Belgium, in 1995 and 2000, respectively. From October 2000 to September 2002, he was at Lucent Technologies, Bell Laboratories, New Jersey, USA, working on the design, processing, and characterization of InP/InGaAsP monolithically integrated devices. In October 2002, he joined the Department of Information Technology (INTEC), Ghent University, Belgium. Currently, he is a member of the permanent staff of the Photonics Group. Since 2008 , he has had a position as a full time professor. He is a lecturer for four courses at Ghent University (Microphotonics, Advanced Photonics Laboratory, Photonic Semiconductor Components, and Technology). He coordinates the cleanroom activities of the research group and is coordinator of the NAMIFAB Center of Expertise. His research focuses on the design, fabrication, and characterization of integrated photonic devices. Main topics involve silicon nanophotonic devices and the integration of innovative materials (III-V, graphene, ferro-electrics, quantum dots, etc.) on these waveguides to expand their functionality. He is working on applications for telecom, diatom, optical interconnect, and sensing. $\mathrm{He}$ has submitted 14 patents, has authored and coauthored more than 220 journal papers, and has presented invited papers at all major conferences in the domain. He is a member of IEEE Photonics Society, OSA, and SPIE. He has coordinated several European Projects (FP6 PICMOS, FP7 WADIMOS, and FP7 SMARTFIBER), contributed in many more, and received an ERC Grant (ULPPIC). He received the prestigious "Laureaat van de Vlaamse Academie Van België" prize in 2012 and was a Clarivate highly cited researcher.

Roel Baets received his MSc degree in electrical engineering from UGent in 1980, his second MSc degree from Stanford in 1981, and his PhD from UGent in 1984. From 1984 to 1989 , he held a postdoctoral position at IMEC. Since 1989, he has been a professor at the Faculty of Engineering and Architecture of UGent where he founded the Photonics Research Group. He is a full professor at Ghent University (UGent) and is associated with IMEC. From 1990 to 1994, he was also a part-time professor at Delft University of Technology and, from 2004 to 2008, at Eindhoven University of Technology. He has mainly worked in the field of integrated photonics. He has made contributions to research on photonic integrated circuits, both in III-V semiconductors and in silicon, as well as their applications in telecom, datacom, sensing, and medicine. Web of Science reports more than 600 publications with an h-index more than 60 . As part of a team of eight professors, he leads the Photonics Research Group. With about 90 researchers, this group is involved in numerous (inter)national research programs and has created four spin-off companies. The silicon photonics activities of the group are part of a joint research initiative with IMEC. He has led major research projects in silicon photonics in Europe. In 2006, he founded ePIXfab, the first Multi-ProjectWafer service for silicon photonics globally. Since then, ePIXfab has evolved to become the European Silicon Photonics Alliance. He is also a director of the multidisciplinary Center for Nano- and Biophotonics (NB Photonics) at UGent founded in 2010. He was a co-founder of the European MSc Program in photonics. He is an ERC grantee of the European Research Council and a Methusalem grantee of the Flemish Government. He is a fellow of the IEEE, European Optical Society (EOS), and Optical Society (OSA). He is also a member of the Royal Flemish Academy of Belgium for Sciences and the Arts. 